\title{
Spontaneous-emission rates in finite photonic crystals of plane scatterers
}

\author{
Martijn Wubs, ${ }^{1,2, *}$ L. G. Suttorp, ${ }^{3}$ and A. Lagendijk ${ }^{1}$ \\ ${ }^{1}$ Complex Photonic Systems, Faculty of Science and Technology, University of Twente, P.O. Box 217, \\ NL-7500 AE Enschede, The Netherlands \\ ${ }^{2}$ Van der Waals-Zeeman Institute, University of Amsterdam, Valckenierstraat 65, NL-1018 XE Amsterdam, The Netherlands \\ ${ }^{3}$ Institute for Theoretical Physics, University of Amsterdam, Valckenierstraat 65, NL-1018 XE Amsterdam, The Netherlands
}

(Received 1 July 2003; published 30 January 2004)

\begin{abstract}
The concept of a plane scatterer that was developed earlier for scalar waves is generalized so that polarization of light is included. Starting from a Lippmann-Schwinger formalism for vector waves, we show that the Green function has to be regularized before $T$ matrices can be defined in a consistent way. After the regularization, optical modes and Green functions are determined exactly for finite structures built up of an arbitrary number of parallel planes, at arbitrary positions, and where each plane can have different optical properties. The model is applied to the special case of finite crystals consisting of regularly spaced identical planes, where analytical methods can be taken further and only light numerical tasks remain. The formalism is used to calculate position- and orientation-dependent spontaneous-emission rates inside and near the finite photonic crystals. The results show that emission rates and reflection properties can differ strongly for scalar and for vector waves. The finite size of the crystal influences the emission rates. For parallel dipoles close to a plane, emission into guided modes gives rise to a peak in the frequency-dependent emission rate.
\end{abstract}

DOI: 10.1103/PhysRevE.69.016616

PACS number(s): 42.70.Qs, 02.30.Rz, 78.66.-w, 42.50.-p

\section{INTRODUCTION}

Photonic crystals are a well-studied subject nowadays, both theoretically and experimentally [1]. Of fundamental importance is the prediction [2] that in three-dimensional photonic crystals that meet a tough combination of requirements, light propagation will be completely inhibited in all directions and a photonic band gap will show up for certain frequencies of light. It is important for technology that photonic crystals can be created that guide light with low losses and bend light on a scale of an optical wavelength. The latter properties do not require a band gap in all three dimensions.

A photonic-band-gap crystal would reflect light for all angles of incidence, when the frequency of the light lies within the gap. However, lower-dimensional photonic crystals such as Bragg mirrors can also be omnidirectional mirrors, without having a band gap [3-5]. Thus, external light sources can only give an indication that there is a band gap or a proof that there is no gap.

Internal light sources such as excited atoms do a better job in probing a band gap, because only a gap would completely inhibit spontaneous emission by internal sources [2]. For the same reason, a photonic-band-gap crystal would be a whole new playground in quantum optics, both when one is interested in spontaneous emission in itself, and in processes which normally are obscured or made less efficient because of spontaneous emission. Not only emission rates would be strongly modified inside a band-gap crystal, but also resonant dipole-dipole interactions, for example, as they are mediated by the electromagnetic field [6]. The focus of this paper is on spontaneous-emission rates of visible light.

For atomic transition frequencies in the band gap of an

\footnotetext{
*Electronic address: c.m.wubs@tn.utwente.nl; URL: http:// tnweb.tn.utwente.nl/cops
}

infinite three-dimensional photonic crystal, emission rates vanish everywhere in the inhomogeneous structure. In practice, such a uniform suppression of emission rates has not yet been observed for visible light: evidence of crystals exhibiting a full photonic band gap in the visible light has not been reported to date. Even when in the future such crystals will exist, position-dependent emission rates will occur at the edges of the crystals. In general, spontaneous-emission rates of inhomogeneous dielectrics with a high refractive-index contrast, including photonic crystals, are strongly position and orientation dependent. Calculated spontaneous-emission rates in this paper will prove this point. Also, finite-size effects will show up in our calculations. The model studied here is a finite photonic crystal consisting of a finite number of parallel and infinitely thin planes. More about our model will be said later in this section.

In many experiments, dipole orientations are hard to control. When averaged over dipole orientations, spontaneousemission rates are proportional to a quantity called the "local optical density of states" (LDOS) [7,8]. The concept of a local density of states was borrowed from solid-state physics. The local optical density of states was first named the "local radiative density of states" [7], which is the same quantity.

Interestingly, the calculation of position-dependent spontaneous-emission rates also has a bearing on the interpretation of measurements performed with a near-field scanning optical microscope. In these measurements, a sample is illuminated through the tip of the microscope, and scattered light is recorded. In a simple model, the disturbance of the optical field by bringing the tip of the microscope to the sample is assumed to be weak, and the tip is modeled as a dipole with a certain strength and orientation. Then, if the light scattered in all directions was recorded, the measured signal would be proportional to the local spontaneousemission rate at the position of the tip in the absence of the tip [9]. 
After the above general considerations, we now turn to the topic of how spontaneous-emission rates inside photonic crystals are actually being calculated. The existence of a band gap in an infinite photonic crystal can be inferred from a band structure calculation, which for a three-dimensional photonic crystals is an art in itself (see the recent review [10]). Quite another and more difficult matter is it to calculate emission rates inside infinite crystals $[11,12]$. Emission rates inside or near finite photonic crystals are even harder to calculate. Other interesting quantities would be near-field or far-field spectra of internal sources, or dipole-dipole interactions and superradiance effects of atoms embedded in a finite three-dimensional photonic crystal, to name a few complex processes in a complex environment. In such cases, results of calculations are hard to check and-even if correct-they might not give much insight.

It is therefore very useful to study complex processes in simplified models for photonic crystals. Widely used is the so-called quasi-one-dimensional model (or isotropic model) for photonic crystals [13], where it is assumed that the red edge of the stop bands of the crystal occur at the same bandedge frequency for all three-dimensional propagation directions, and similarly for the blue band edge. Such a model will describe qualitatively correct the processes well inside the band gap, while overestimating effects of the photonic crystal at the edges of the gap. The isotropic model also neglects all position and orientation dependence of emission rates outside the band gap. Inspired by the model calculations, more realistic numerical calculations have recently appeared that indeed show the weaknesses of the isotropic model [12].

In this paper another simple model is proposed, one which takes into account the strong spatial and orientational dependence of optical properties and the finite size of the crystals. On the other hand, it gives up the existence of a full band gap, as only variations of the refractive index in one dimension are considered. Dielectric slabs are modeled as infinitely thin planes, which will be called plane scatterers. A multiplescattering formalism is set up in which optical modes and the Green function (a tensor, really) can be calculated exactly for crystals consisting of an arbitrary number of plane scatterers. The present model is a generalization of previous work that treated scalar waves only [5]. The inclusion of polarization of light will turn out not to be straightforward.

Infinitely thin planes were used as model systems in photonics before, for example, in Ref. [14] where light propagation was considered in one dimension only and for an infinite crystal. The model was generalized in Ref. [15], where infinite photonic crystals were built of infinitely thin planes and their band structure was determined for waves propagating in three dimensions. In Ref. [16], both infinite and finite crystals of planes were considered and their transmission and reflection properties were studied with the use of transfermatrix methods. The infinite crystal was again considered in Refs. [17-20] and named the "Dirac-comb superlattice." Frequency-dependent emission rates were determined for several positions in the unit cell and both TE $[17,19]$ and TM waves $[18,20]$ were considered. The periodicity of infinite crystals gives that Bloch's theorem can be used in the analy- sis. Finite photonic crystals do not have this advantage and the analysis of the optical properties is usually more difficult. Position-dependent spontaneous-emission rates remain to be explored in model photonic crystals consisting of a finite number of plane scatterers, where light propagation in all three dimensions is taken into account, for both polarization directions.

It has been known for a long time that spontaneousemission rates of atoms change when positioned at distances of the order of the wavelength of light away from a mirror [21-23]. A recent surprise was the measurement and analysis that even a distant mirror ( $25 \mathrm{~cm}$ away) can change emission rates when lenses are used and when the atomic positions are controlled with a subwavelength precision (a few nanometers) [24,25].

More complicated than emission near a mirror is it to calculate emission rates of atoms in or near one-dimensional photonic crystals. A multipurpose formalism for calculating optical modes in layered dielectrics [26] was used in Ref. [4] to calculate emission rates inside finite periodic layered structures, especially inside structures that reflect light incoming from all directions, the so-called "omnidirectional mirrors" [3].

Interestingly, light transmission through finite onedimensional photonic crystals can be found exactly in terms of the transmission through a unit cell, the number $N$ of unit cells, and in terms of the Bloch wave vector of the corresponding infinite-crystal structure. In Ref. [27], this is shown for a simple unit cell containing two layers, but it was also proven for general unit cells [28]. This remarkable result was reviewed in Ref. [29], where its importance is stressed not only in optics but also in acoustics, quantum mechanics, and other branches of physics. In the $T$-matrix formalism of this paper (which differs from the usual transfer-matrix method for layered dielectrics), we find similar analytical results, also involving the Bloch wave vector. Such attractive analytical results are not available for more complex dielectrics such as finite two- $[30,31]$ or three-dimensional photonic crystals [32], so that in those cases the use of efficient numerical techniques is essential.

The advantage of our plane-scatterer model is that modes and Green functions (and therefore emission rates) can be calculated exactly in a Lippmann-Schwinger formalism, for every finite-crystal size, and that light propagation in all directions is taken into account. Lippmann-Schwinger formalisms are more commonly used [33], but when the finite volumes of dielectric scatterers are fully taken into account, numerical discretization of the dielectric is required and the model stops being simple [31,34]. To be sure, the simplicity of our model entails that in some aspects it becomes less realistic, as will be stressed where appropriate.

In Sec. II, multiple scattering of light is introduced and central equations are derived in representation-independent notation. In Sec. III the free-space Green tensor is regularized and a $T$ matrix of a plane scatterer for light waves is derived. Section IV discusses all optical modes (propagating and guided modes, including polarization) that exist in crystals of plane scatterers. Position- and orientation-dependent 
spontaneous-emission rates are calculated in Sec. V. Conclusions can be found in Sec. VI.

\section{MULTIPLE-SCATTERING THEORY FOR VECTOR WAVES}

Some important equations of multiple-scattering theory [33] will be presented, mostly in representation-independent notation, for light in arbitrary inhomogeneous dielectrics. In later sections a particular class of dielectrics will be studied and a suitable representation is chosen, but then the involved notation might obscure the general structure of the equations.

The wave equation for the electric field $\mathbf{E}_{0}(\mathbf{r}, \omega)$ in free space is

$$
\begin{gathered}
(\omega / c)^{2} \mathbf{E}_{0}(\mathbf{r}, \omega)-\nabla \times \nabla \times \mathbf{E}_{0}(\mathbf{r}, \omega)=0 \Leftrightarrow \\
\left\{(\omega / c)^{2} \mathbf{I} \cdot-\boldsymbol{\nabla} \times \boldsymbol{\nabla} \times\right\} \mathbf{E}_{0}(\mathbf{r}, \omega)=\mathbf{0} .
\end{gathered}
$$

The symbol I denotes the unit tensor in three-dimensional space. The solutions of Eq. (1a) are plane waves with wave vector $\mathbf{k}$ and polarization direction normal to $\mathbf{k}$. With the free-space wave equation (1a), a Green tensor (or dyadic Green function) is associated that satisfies

$$
\left\{(\omega / c)^{2} \mathbf{I} \cdot-\boldsymbol{\nabla} \times \boldsymbol{\nabla} \times\right\} \mathbf{G}_{0}\left(\mathbf{r}, \mathbf{r}^{\prime}, \omega\right)=\delta^{3}\left(\mathbf{r}-\mathbf{r}^{\prime}\right) \mathbf{I} .
$$

Let $\mathbf{L}(\mathbf{r}, \omega)$ be the quantity between curly brackets in Eqs. (1b) and (2). Both equations can be considered as the realspace representations of an abstract tensor operator $\mathbf{L}(\omega)$ operating on the vector field $\mathbf{E}_{0}(\omega)$ and on the Green function $\mathbf{G}_{0}(\omega)$, respectively,

$$
\mathbf{L}(\omega) \cdot \mathbf{E}_{0}(\omega)=0, \quad \mathbf{L}(\omega) \cdot \mathbf{G}_{0}(\omega)=1 \otimes \mathbf{I}
$$

The identity operator in real space is denoted by $\mathbb{l}$ and it has the property $\left\langle\mathbf{r}|\mathbb{I}| \mathbf{r}^{\prime}\right\rangle=\delta^{3}\left(\mathbf{r}-\mathbf{r}^{\prime}\right)$; confusion with the unit tensor I should not arise; $\otimes$ denotes the tensor product.

In the presence of an inhomogeneous dispersive linear dielectric, the wave equation for the electric field is modified into

$$
\mathbf{L}(\omega) \cdot \mathbf{E}(\omega)=\mathbf{V}(\omega) \cdot \mathbf{E}(\omega)
$$

where the frequency-dependent optical potential $\mathbf{V}$ is defined in terms of the dielectric function $\varepsilon(\mathbf{r}, \omega)$ as

$$
\left\langle\mathbf{r}|\mathbf{V}(\omega)| \mathbf{r}^{\prime}\right\rangle=-[\varepsilon(\mathbf{r}, \omega)-1](\omega / c)^{2} \mathbf{I} \delta\left(\mathbf{r}-\mathbf{r}^{\prime}\right)
$$

The $\delta$-function on the right-hand side defines the potential as a local quantity (which the $T$ matrix, to be defined shortly, is not). In other words, this $\delta$ function appears for any potential.

The electric field $\mathbf{E}_{0}(\omega)$ is modified into $\mathbf{E}(\omega)$, and the two fields are related through the Lippmann-Schwinger (LS) equation

$$
\begin{aligned}
\mathbf{E}(\omega)= & \mathbf{E}_{0}(\omega)+\mathbf{G}_{0}(\omega) \cdot \mathbf{V}(\omega) \cdot \mathbf{E}(\omega) \\
= & \mathbf{E}_{0}(\omega)+\mathbf{G}_{0}(\omega) \cdot \mathbf{V}(\omega) \cdot \mathbf{E}_{0}(\omega) \\
& +\mathbf{G}_{0}(\omega) \cdot \mathbf{V}(\omega) \cdot \mathbf{G}_{0}(\omega) \cdot \mathbf{V}(\omega) \cdot \mathbf{E}_{0}(\omega)+\cdots \\
= & \mathbf{E}_{0}(\omega)+\mathbf{G}_{0}(\omega) \cdot \mathbf{T}(\omega) \cdot \mathbf{E}_{0}(\omega) .
\end{aligned}
$$

One can check that indeed the field $\mathbf{E}(\omega)$ that satisfies Eq. (6a) is also a solution of Eq. (4). The solution of Eq. (6a) can be found iteratively in higher and higher orders of the optical potential V, as given by the multiple-scattering series in Eq. (6b); the (dyadic) $T$ matrix in Eq. (6c) by definition is the formal sum of the infinite summation in Eq. (6b). The $T$ matrix is a $3 \times 3$ tensor. By combining Eqs. (6b) and (6c), the formal solution for the $T$ matrix is

$$
\mathbf{T}(\omega)=\mathbf{V}(\omega) \cdot\left[1 \otimes \mathbf{I}-\mathbf{G}_{0}(\omega) \cdot \mathbf{V}(\omega)\right]^{-1}
$$

The scattering problem is solved exactly once the $T$ matrix is known.

There may exist optical modes that are bound to the scatterer. Such bound modes correspond to solutions of the LS equation (6a) in the absence of an incident field; with Eq. (7) we can rewrite this homogeneous equation as

$$
\mathbf{T}^{-1}(\omega) \cdot \mathbf{V}(\omega) \cdot \mathbf{E}(\omega)=\mathbf{0}
$$

It follows that bound solutions of the electric field will correspond to the poles of the $T$ matrix. Actually, Eq. (6c) also shows that a nonzero solution for $\mathbf{E}(\omega)$ can only occur when $\mathbf{T}(\omega)$ has a pole. The $T$ matrix not only solves the scattering problem for incident fields but also contains all information about bound modes.

In the presence of the dielectric the Green function also changes, from $\mathbf{G}_{0}$ to $\mathbf{G}$. The latter satisfies

$$
[\mathbf{L}(\omega)-\mathbf{V}(\omega)] \cdot \mathbf{G}(\omega)=1 \otimes \mathbf{I}
$$

The solution for the Green function analogous to Eq. (6) for the electric field is the three-dimensional Dyson-Schwinger equation

$$
\begin{aligned}
\mathbf{G}(\omega) & =\mathbf{G}_{0}(\omega)+\mathbf{G}_{0}(\omega) \cdot \mathbf{V}(\omega) \cdot \mathbf{G}(\omega) \\
& =\mathbf{G}_{0}(\omega)+\mathbf{G}_{0}(\omega) \cdot \mathbf{T}(\omega) \cdot \mathbf{G}_{0}(\omega) .
\end{aligned}
$$

It can be verified that a solution of Eq. (10a) also is a solution of Eq. (9). The problem of how to find such a solution is solved once the $T$ matrix (7) is determined, because an iteration of Eq. (10a) analogous to the series expansion (6b) for the electric field shows that the Green function can also be expressed in terms of the $T$ matrix, as given by Eq. (10b).

Equation (10) also holds when the total potential $\mathbf{V}(\omega)$ is a sum of single-scatterer potentials $\mathbf{V}_{\alpha}(\omega)$. By iterating one finds that the total $T$ matrix for an arbitrary number $N$ of these scatterers is 


$$
\begin{aligned}
\mathbf{T}^{(N)}= & \sum_{\alpha=1}^{N} \mathbf{V}_{\alpha}+\sum_{\alpha, \beta} \mathbf{V}_{\beta} \cdot \mathbf{G}_{0} \cdot \mathbf{V}_{\alpha}+\sum_{\alpha, \beta, \gamma} \mathbf{V}_{\gamma} \cdot \mathbf{G}_{0} \cdot \mathbf{V}_{\beta} \cdot \mathbf{G}_{0} \cdot \mathbf{V}_{\alpha} \\
& +\cdots
\end{aligned}
$$

Often it is more convenient to make an equivalent expansion in terms of the single-scatterer $T$ matrices [33]:

$$
\begin{aligned}
\mathbf{T}^{(N)}= & \sum_{\alpha=1}^{N} \mathbf{T}_{\alpha}+\sum_{\alpha, \beta(\neq \alpha)} \mathbf{T}_{\beta} \cdot \mathbf{G}_{0} \cdot \mathbf{T}_{\alpha} \\
& +\sum_{\alpha, \beta(\neq \alpha), \gamma(\neq \beta)} \mathbf{T}_{\gamma} \cdot \mathbf{G}_{0} \cdot \mathbf{T}_{\beta} \cdot \mathbf{G}_{0} \cdot \mathbf{T}_{\alpha}+\cdots
\end{aligned}
$$

The frequency dependence was suppressed in Eqs. (11) and (12). The form of Eq. (12) of the total $T$ matrix will be used later in this paper, for model systems where the infinite summation can be performed explicitly.

\section{PLANE SCATTERERS FOR VECTOR WAVES}

The general results of multiple-scattering theory that were presented in Sec. II will now be applied to dielectrics that can be described as a collection of parallel planes. A suitable representation is chosen, and specific forms of the potential $\mathbf{V}$, the free-space Green function $\mathbf{G}_{0}$, and the incoming electric field $\mathbf{E}_{0}$ are determined. With this, $T$ matrices for a single plane and for an arbitrary number of planes are derived.

\section{A. Dyadic Green function in plane representation}

A solution for the free-space dyadic Green function can be found in three-dimensional Fourier space. By translational invariance, $\left\langle\mathbf{k}\left|\mathbf{G}_{0}(\omega)\right| \mathbf{k}^{\prime}\right\rangle$ must be equal to $(2 \pi)^{3} \delta^{3}(\mathbf{k}$ $\left.-\mathbf{k}^{\prime}\right) \mathbf{G}_{0}(\mathbf{k}, \omega)$. The Green function $\mathbf{G}_{0}(\mathbf{k}, \omega)$ satisfies

$$
\left\{\left[(\omega / c)^{2}-k^{2}\right] \mathbf{I}+k^{2} \hat{\mathbf{k}} \hat{\mathbf{k}}\right\} \cdot \mathbf{G}_{0}(\mathbf{k}, \omega)=\mathbf{I} .
$$

Here, $\hat{\mathbf{k}}$ denotes a unit vector in the direction of the wave vector $\mathbf{k}$. Equation (13) is a $3 \times 3$ matrix equation whose representation diagonalizes in the polarization basis $\left\{\hat{\mathbf{k}}, \hat{\boldsymbol{\sigma}}_{1}, \hat{\boldsymbol{\sigma}}_{2}\right\}$ with the longitudinal direction $\hat{\mathbf{k}}$ and two orthogonal transverse directions $\hat{\boldsymbol{\sigma}}_{1,2}$. The solution of Eq. (13) is

$$
G_{0}^{j j}(\mathbf{k}, \omega)=\frac{1}{(\omega / c)^{2}-k^{2}}, \quad G_{0}^{\hat{\mathbf{k}} \hat{\mathbf{k}}}(\mathbf{k}, \omega)=(c / \omega)^{2},
$$

where $j$ denotes $\sigma_{1}$ or $\sigma_{2}$. All six nondiagonal elements of the Green tensor are zero in this representation. This is the retarded Green function once we assume that the frequency $\omega$ has an infinitesimally small positive imaginary part.

The above Fourier representation is not what we need. It is convenient to work in the "plane representation:" in twodimensional Fourier space in the directions parallel to the planes and in real space in the $\hat{\mathbf{z}}$ direction perpendicular to the planes. For the polarization representation choose the orthonormal basis $\left\{\hat{\mathbf{s}}_{\mathbf{k}}, \hat{\mathbf{v}}_{\mathbf{k}}, \hat{\mathbf{z}}\right\}$. Here, $\hat{\mathbf{z}}$ is the unit vector in the $z$ direction; $\hat{\mathbf{v}}_{\mathbf{k}}$ is the unit vector in the direction of the projection of the wave vector $\mathbf{k}$ on the plane, so that the wave vector $\mathbf{k}$ has a component $k_{\|}$in the $\hat{\mathbf{v}}_{\mathbf{k}}$ direction and its full representation is $\left(0, k_{\|}, k_{z}\right)$; the $s_{\mathbf{k}}$-polarization direction is orthogonal to the optical plane that is spanned by the other two basis vectors. Then the operator $\mathbf{L}(\omega)$ has the form

$$
\left\langle\mathbf{k}_{\|}, z|\mathbf{L}(\omega)| \mathbf{k}_{\|}^{\prime}, z^{\prime}\right\rangle=(2 \pi)^{2} \delta^{2}\left(\mathbf{k}_{\|}-\mathbf{k}_{\|}^{\prime}\right) \delta\left(z-z^{\prime}\right) \mathbf{L}\left(\mathbf{k}_{\|}, z, \omega\right),
$$

where the operator $\mathbf{L}\left(\mathbf{k}_{\|}, z, \omega\right)$ has the matrix representation

$$
\left(\begin{array}{ccc}
(\omega / c)^{2}-k_{\|}^{2}+\partial_{z}^{2} & 0 & 0 \\
0 & (\omega / c)^{2}+\partial_{z}^{2} & -i k_{\|} \partial_{z} \\
0 & -i k_{\|} \partial_{z} & (\omega / c)^{2}-k_{\|}^{2}
\end{array}\right) .
$$

The Green function in the same representation becomes $\left\langle\mathbf{k}_{\|}, z\left|\mathbf{G}_{0}(\omega)\right| \mathbf{k}_{\|}^{\prime}, z^{\prime}\right\rangle=(2 \pi)^{2} \delta^{2}\left(\mathbf{k}_{\|}-\mathbf{k}_{\|}^{\prime}\right) \mathbf{G}_{0}\left(\mathbf{k}_{\|}, z, z^{\prime}, \omega\right)$, and the transformed Eq. (13) is a system of differential equations:

$$
\mathbf{L}\left(\mathbf{k}_{\|}, z, \omega\right)\left(\begin{array}{ccc}
G_{0}^{s s} & G_{0}^{s v} & G_{0}^{s z} \\
G_{0}^{v s} & G_{0}^{v v} & G_{0}^{v z} \\
G_{0}^{z s} & G_{0}^{z v} & G_{0}^{z z}
\end{array}\right)=\delta\left(z-z^{\prime}\right)\left(\begin{array}{ccc}
1 & 0 & 0 \\
0 & 1 & 0 \\
0 & 0 & 1
\end{array}\right) .
$$

$G_{0}^{p q}$ are the components of $\mathbf{G}_{0}$ and their arguments $\left(\mathbf{k}_{\|}, z, z^{\prime}, \omega\right)$ were dropped for brevity. By choosing the plane representation, the matrix elements of $\mathbf{G}_{0}$ only depend on the magnitude and not on the orientation of $\mathbf{k}_{\|}$. All components involving an $s$ label are zero, except the $s s$ component. $G_{0}^{s s}$ satisfies the same differential equation as the Green function $g_{0}$ of the Helmholtz equation for scalar waves, so that for $\omega>0$ we have

$$
G_{0}^{s s}\left(\mathbf{k}_{\|}, z, z^{\prime}, \omega\right)=g_{0}\left(\mathbf{k}_{\|}, z, z^{\prime}, \omega\right)=\frac{e^{i k_{z}\left|z-z^{\prime}\right|}}{2 i k_{z}} .
$$

The variable $k_{z}$ is not independent of $\mathbf{k}_{\|}$, but rather an abbreviation for $\left[(\omega / c)^{2}-k_{\|}^{2}\right]^{1 / 2}$. The remaining coupled differential equations of Eq. (16) can also be solved (again for $\omega>0$ ), now that $G_{0}^{s s}$ is known:

$$
\begin{gathered}
G_{0}^{v v}\left(\mathbf{k}_{\|}, z, z^{\prime}, \omega\right)=\frac{k_{z}^{2}}{(\omega / c)^{2}} g_{0}, \\
G_{0}^{v z}\left(\mathbf{k}_{\|}, z, z^{\prime}, \omega\right)=-\frac{k_{\|} k_{z}}{(\omega / c)^{2}} g_{0} \operatorname{sgn}\left(z-z^{\prime}\right), \\
G_{0}^{z v}\left(\mathbf{k}_{\|}, z, z^{\prime}, \omega\right)=G_{0}^{v z}, \\
G_{0}^{z z}\left(\mathbf{k}_{\|}, z, z^{\prime}, \omega\right)=\frac{1}{(\omega / c)^{2}}\left[k_{\|}^{2} g_{0}+\delta\left(z-z^{\prime}\right)\right] .
\end{gathered}
$$

Green functions in the right-hand sides are understood to have the arguments $\left(\mathbf{k}_{\|}, z, z^{\prime}, \omega\right)$. The above method of solving differential equations does not give a value for the 
signum function when $z$ is equal to $z^{\prime}$. The Green-function components (18) can alternatively be found from an inverse Fourier transformation

$$
\mathbf{G}_{0}\left(\mathbf{k}_{\|}, z, z^{\prime}, \omega\right)=\frac{1}{2 \pi} \int_{-\infty}^{\infty} d k_{z}^{\prime} \mathbf{G}_{0}\left(\mathbf{k}_{\|}, k_{z}^{\prime}, \omega\right) e^{i k_{z}^{\prime}\left(z-z^{\prime}\right)} .
$$

This integration can only be performed in a representation that does not corotate with $k_{z}^{\prime}$. The basis of Eq. (14) is not adequate, but again the basis $\left\{\hat{\mathbf{s}}_{\mathbf{k}}, \hat{\mathbf{v}}_{\mathbf{k}}, \hat{\mathbf{z}}\right\}$ suits well. With this Fourier method one finds the value 0 for the signum function in Eq. (18b) when $z$ equals $z^{\prime}$ : for $z=z^{\prime}$ the relevant integrands in Eq. (19) are antisymmetric in the variable $k_{z}^{\prime}$.

\section{B. Regularization of the Green function}

The $T$ matrix of a plane scatterer for vector waves can be found by solving the appropriate Lippmann-Schwinger equation (6). A plane wave incident from $z=-\infty$ with wave vector $\mathbf{k}$ and arbitrary amplitude $E_{0}$ and transverse polarization vector $\boldsymbol{\sigma}_{\mathbf{k}}=\left(\sigma_{s}, \sigma_{v}, \sigma_{z}\right)$ is scattered by a plane at $z=z_{\alpha}$. Because of the symmetry in the in-plane directions, it is convenient to choose the plane representation for the LS equation. In terms of the Dirac notation, the electric field is a "ket;" the plane representation is found by taking the inner product of Eq. (6) for the electric field with the "bra" $\left\langle\mathbf{k}_{\|}, z\right|$, and by inserting the unit operator

$$
\frac{1}{(2 \pi)^{2}} \int d^{2} \mathbf{k}_{\|}^{\prime} d z^{\prime}\left|\mathbf{k}_{\|}^{\prime}, z^{\prime}\right\rangle\left\langle\mathbf{k}_{\|}^{\prime}, z^{\prime}\right|
$$

at the positions of the dots in the representation-independent equation (6). The incident field takes the form $\mathbf{E}_{\mathbf{k} \boldsymbol{\sigma}, 0}\left(\mathbf{k}_{\|}, z, \omega\right)=E_{0} \boldsymbol{\sigma}_{\mathbf{k}} \exp \left(i k_{z} z\right)$. The solution of the LS equation corresponding to this incident field is $\mathbf{E}_{\mathbf{k} \boldsymbol{\sigma}}(\omega)$. The LS equation in the mixed representation becomes

$$
\begin{aligned}
\mathbf{E}_{\mathbf{k} \boldsymbol{\sigma}}\left(\mathbf{k}_{\|}, z, \omega\right) & \\
= & E_{0} \boldsymbol{\sigma}_{\mathbf{k}} e^{i k_{z} z} \\
& \quad+\int_{-\infty}^{+\infty} d z^{\prime} \mathbf{G}_{0}\left(\mathbf{k}_{\|}, z, z^{\prime}, \omega\right) \cdot \mathbf{V}\left(z^{\prime}, \omega\right) \cdot \mathbf{E}_{\mathbf{k} \boldsymbol{\sigma}}\left(\mathbf{k}_{\|}, z^{\prime}, \omega\right) .
\end{aligned}
$$

A plane is assumed to be infinitely thin and it can be described by the optical potential $\mathbf{V}(z, \omega)=V(\omega) \delta\left(z-z_{\alpha}\right) \mathbf{I}$. (A specific model potential will be chosen in Sec. III F.) The integral can be evaluated immediately and we get

$$
\begin{aligned}
\mathbf{E}_{\mathbf{k} \boldsymbol{\sigma}}\left(\mathbf{k}_{\|}, z, \omega\right)= & E_{0} \boldsymbol{\sigma}_{\mathbf{k}} e^{i k_{z} z} \\
& +V(\omega) \mathbf{G}_{0}\left(\mathbf{k}_{\|}, z, z_{\alpha}, \omega\right) \cdot \mathbf{E}_{\mathbf{k} \boldsymbol{\sigma}}\left(\mathbf{k}_{\|}, z_{\alpha}, \omega\right) .
\end{aligned}
$$

The usual way to solve this equation would be to put the position $z$ equal to $z_{\alpha}$ and to solve for $\mathbf{E}_{\mathbf{k} \boldsymbol{\sigma}}\left(\mathbf{k}_{\|}, z_{\alpha}, \omega\right)$. The result would then be inserted back into the above equation to obtain an expression for $\mathbf{E}_{\mathbf{k} \boldsymbol{\sigma}}\left(\mathbf{k}_{\|}, z, \omega\right)$.
However, the Green tensor $\mathbf{G}_{0}$ is not defined when the positions $z$ and $z_{\alpha}$ are identical, because of the $\delta$ function in the component $G_{0}^{z z}$ [Eq. (18d)]. One could just neglect the $\delta$ function, as might be correct in other situations [35], but it will be argued in Sec. III C that this procedure would be wrong in our case. Therefore, the Green tensor (18) is not suited for setting up a theory for the scattering of vector waves by infinitely thin planes.

It is known that "regularization" of Green functions is sometimes needed when modeling finite-sized scatterers as mathematical objects with zero volume, in order to have a model that is relevant for optics. (Regularization was not needed for scalar waves scattering off planes [5].) In a regularization procedure, usually a cutoff parameter is introduced that modifies the behavior of Green functions at distances much smaller than optical wavelengths, and mathematical problems are thus overcome. In some cases, the regularization parameter can be sent to infinity in the final stage, while in other cases the cutoff parameter must be kept finite. For example, for point scatterers the problem of diverging Green functions occurs both for scalar and for vector waves. Point scatterers have been studied extensively and several regularization schemes have been proposed (see Ref. [36] and references therein).

The same regularization procedure will now be chosen for plane scatterers as was done before for point scatterers [36]. A high-momentum cutoff is introduced in three-dimensional Fourier space. Instead of the free-space Green function $\mathbf{G}_{0}(\mathbf{k}, \omega)$ of Eq. (14), a regularized free-space Green function $\tilde{\mathbf{G}}_{0}(\mathbf{k}, \omega)$ will be used. The latter is defined in terms of the former as

$$
\tilde{\mathbf{G}}_{0}(\mathbf{k}, \omega)=\left(\frac{\Lambda^{2}}{\Lambda^{2}+k^{2}}\right) \mathbf{G}_{0}(\mathbf{k}, \omega) .
$$

The cutoff momentum $\Lambda$ is assumed to be much larger than the magnitude $\omega / c$ of the optical momentum, so that at optical wavelengths $\tilde{\mathbf{G}}_{0} \simeq \mathbf{G}_{0}$. The effect of this cutoff in the real-space representation is also known [36]. Here its effect on the Green function in the plane representation is important. After an inverse Fourier transformation, again only in the $z$ direction, one obtains (again for $\omega>0$ )

$$
\begin{gathered}
\widetilde{G}_{0}^{s s}\left(\mathbf{k}_{\|}, z, z_{1}, \omega\right)=\frac{\Lambda^{2}}{\Lambda^{2}+(\omega / c)^{2}}\left(g_{0}+\frac{e^{-\Lambda_{\|}\left|z-z_{1}\right|}}{2 \Lambda_{\|}}\right), \\
\widetilde{G}_{0}^{v v}\left(\mathbf{k}_{\|}, z, z_{1}, \omega\right)=\frac{k_{z}^{2} c^{2}}{\omega^{2}} \widetilde{G}_{0}^{s s}, \\
\widetilde{G}_{0}^{v z}\left(\mathbf{k}_{\|}, z, z_{1}, \omega\right)=-\frac{\Lambda^{2} \operatorname{sgn}\left(z-z_{1}\right)}{\Lambda^{2}+(\omega / c)^{2}} \frac{k_{\|}}{(\omega / c)^{2}} \\
\times\left[k_{z} g_{0}+(i / 2) e^{-\Lambda \|\left|z-z_{1}\right|}\right], \\
\widetilde{G}_{0}^{z v}\left(\mathbf{k}_{\|}, z, z_{1}, \omega\right)=\widetilde{G}_{0}^{v z},
\end{gathered}
$$




$$
\begin{aligned}
\widetilde{G}_{0}^{z z}\left(\mathbf{k}_{\|}, z, z_{1}, \omega\right)= & \frac{\Lambda^{2}}{\Lambda^{2}+(\omega / c)^{2}}\left[\frac{k_{\|}^{2} c^{2}}{\omega^{2}} g_{0}\right. \\
& \left.+\frac{\Lambda_{\|}^{2}+(\omega / c)^{2}}{2 \Lambda_{\|}(\omega / c)^{2}} e^{-\Lambda_{\|}\left|z-z_{1}\right|}\right]
\end{aligned}
$$

In the right-hand sides, the arguments $\left(\mathbf{k}_{\|}, z, z_{1}, \omega\right)$ of the Green functions were dropped; $\Lambda_{\|}$is short-hand notation for $\left(\Lambda^{2}+k_{\|}^{2}\right)^{1 / 2}$; again, the signum function is zero when its argument is.

All components of the regularized Green tensor consist of two parts: an oscillating and a decaying part, as a function of $\left|z-z_{1}\right|$. The decay occurs at a distance that is a tiny fraction of an optical wavelength. For $\Lambda\left|z-z_{1}\right| \gtrsim 1$, the regularized Green function approaches the unregularized one. If one would take the limit $\Lambda \rightarrow \infty$, then all the components in Eq. (24) approach the unregularized components of Eq. (18), and in particular the limit of the last term in $\mathbf{G}_{0}^{z z}$ gives the $\delta$ function that made the regularization procedure necessary. However, $\Lambda$ is kept finite for the moment, so that $\widetilde{G}_{0}^{z z}$ has a finite term that grows with $\Lambda$. With this result, the Greenfunction regularization is complete and a theory of scattering by vector waves from plane scatterers can be set up.

\section{C. $T$ matrix of a plane for vector waves}

The regularization entails that the Green function is replaced by its regularized version in the LS equation (21). For $z=z_{\alpha}$ that equation becomes

$$
\tilde{\mathbf{T}}\left(\mathbf{k}_{\|}, \omega\right)=\mid \begin{gathered}
\frac{V(\omega)}{1-V(\omega) \widetilde{G}_{0}^{s s}} \\
0 \\
0
\end{gathered}
$$

The scattering of the $s$-polarization component of the light can be considered independently from the $\hat{\mathbf{v}}$ and $\hat{\mathbf{z}}$ directions, according to Eqs. (26) and (27). It can be verified with Eqs. (24) and (27) that, since $\Lambda \gg(\omega / c)$, the matrix component $\widetilde{T}^{s s}$ for all practical purposes is equal to the $T$ matrix for scalar waves, and the same holds for the Green tensor component $\widetilde{G}_{0}^{s s}$ : the regularization was not necessary for $s$-polarized light and fortunately it does not affect the scattering properties of $s$-polarized light.

The need for regularization did show up in the description of scattering of $p$-polarized light, and there the cutoff might

$$
\begin{aligned}
& \left(\begin{array}{c}
E_{\mathbf{k} \boldsymbol{\sigma}}^{s}\left(\mathbf{k}_{\|}, z_{\alpha}, \omega\right) \\
E_{\mathbf{k} \boldsymbol{\sigma}}^{v}\left(\mathbf{k}_{\|}, z_{\alpha}, \omega\right) \\
E_{\mathbf{k} \boldsymbol{\sigma}}^{z}\left(\mathbf{k}_{\|}, z_{\alpha}, \omega\right)
\end{array}\right) \\
& \quad=E_{0}\left(\begin{array}{c}
\sigma_{s} \\
\sigma_{v} \\
\sigma_{z}
\end{array}\right) e^{i k_{z} z_{\alpha}+V(\omega)}\left(\begin{array}{ccc}
\widetilde{G}_{0}^{s s} & 0 & 0 \\
0 & \widetilde{G}_{0}^{v v} & 0 \\
0 & 0 & \widetilde{G}_{0}^{z z}
\end{array}\right) \\
& \quad \times\left(\begin{array}{c}
E_{\mathbf{k} \boldsymbol{\sigma}}^{s}\left(\mathbf{k}_{\|}, z_{\alpha}, \omega\right) \\
E_{\mathbf{k} \boldsymbol{\sigma}}^{v}\left(\mathbf{k}_{\|}, z_{\alpha}, \omega\right) \\
E_{\mathbf{k} \boldsymbol{\sigma}}^{z}\left(\mathbf{k}_{\|}, z_{\alpha}, \omega\right)
\end{array}\right) .
\end{aligned}
$$

Here $\widetilde{G}_{0}^{s s}$ stands for $\widetilde{G}_{0}^{s s}\left(\mathbf{k}_{\|}, z_{\alpha}, z_{\alpha}, \omega\right)$, and similarly for the other components. The off-diagonal elements of the Green tensor are all zero when the position $z$ is equal to $z_{\alpha}$, so that the equation can be solved for every component separately. By inserting this result into the LS equation for general $z$, one finds

$$
\begin{aligned}
\mathbf{E}_{\mathbf{k} \boldsymbol{\sigma}}\left(\mathbf{k}_{\|}, z, \omega\right)= & \mathbf{E}_{\mathbf{k} \boldsymbol{\sigma}, 0}\left(\mathbf{k}_{\|}, z, \omega\right) \\
& +\tilde{\mathbf{G}}_{0}\left(\mathbf{k}_{\|}, z, z_{\alpha}, \omega\right) \cdot \tilde{\mathbf{T}}\left(\mathbf{k}_{\|}, \omega\right) \cdot \mathbf{E}_{\mathbf{k} \boldsymbol{\sigma}, 0}\left(\mathbf{k}_{\|}, z_{\alpha}, \omega\right),
\end{aligned}
$$

where the $T$ matrix for scattering from a plane by arbitrarily polarized light is given by

influence light scattering. Incoming $p$-polarized light is characterized by its amplitude $E_{0}$, wave vector $\mathbf{k}$, and its polarization state $\hat{\boldsymbol{\sigma}}=\hat{p} \equiv\left(k_{z} / k\right) \hat{\mathbf{v}}_{\mathbf{k}}-\left(k_{\|} / k\right) \hat{\mathbf{z}}$. Written out explicitly, the incoming field is $\mathbf{E}_{\mathbf{k} \boldsymbol{\sigma}, 0}\left(\mathbf{k}_{\|}, z, \omega\right)$ $=E_{0}\left(0, k_{z} / k,-k_{\|} / k\right) \exp \left(i k_{z} z\right)$. For distances far enough from the plane so that $\Lambda\left|z-z_{\alpha}\right| \gg 1$, the term $\widetilde{G}_{0}^{v z} \widetilde{T}^{z z}$ in Eq. (26) falls off as $\Lambda^{-1}$ and $\widetilde{G}_{0}^{z z} \widetilde{T}^{z z}$ as $\exp \left(-\Lambda_{\|}\left|z-z_{\alpha}\right|\right)$, so that for optical purposes these terms can be neglected. For finite very large $\Lambda$ we arrive at the following effective description: 


$$
\begin{aligned}
\left(\begin{array}{c}
E^{s}(z) \\
E^{v}(z) \\
E^{z}(z)
\end{array}\right)= & \left(\begin{array}{c}
E_{0}^{v}(z) \\
E_{0}^{v}(z) \\
E_{0}^{z}(z)
\end{array}\right)+\left(\begin{array}{ccc}
G_{0}^{s s} & 0 & 0 \\
0 & G_{0}^{v v} & G_{0}^{v z} \\
0 & G_{0}^{z v} & G_{0}^{z z}
\end{array}\right) \\
& \times\left(\begin{array}{ccc}
T^{s s} & 0 & 0 \\
0 & T^{v v} & 0 \\
0 & 0 & 0
\end{array}\right)\left(\begin{array}{c}
E_{0}^{s}\left(z_{\alpha}\right) \\
E_{0}^{v}\left(z_{\alpha}\right) \\
E_{0}^{z}\left(z_{\alpha}\right)
\end{array}\right),
\end{aligned}
$$

where the $s s$-component of the $T$ matrix is equal to $V(\omega)\left[1-V(\omega) G_{0}^{s s}\right]^{-1}$, and analogously for the $v v$-component. The Green functions have arguments $\left(\mathbf{k}_{\|}, z, z_{\alpha}, \omega\right)$. In this effective description-where the $T$ matrix is denoted by $\mathbf{T}$ rather than $\tilde{\mathbf{T}}$ - the cutoff parameter $\Lambda$ does not occur anymore. The cutoff was necessary in order to set up a scattering theory and it shows up in the elements of the scattering theory such as the $T$ matrix (27) and the regularized Green function (24). It does not show up in the electric field, and precisely this enables us to arrive at the effective description. Note also that the (large) value of $G_{0}^{z z}$ has become irrelevant.

The effective description that is obtained here after a regularization is different from a theory where the $\delta$ function in $G_{0}^{z z}$ [see Eq. (18d)] would simply be removed [35]. Leaving out the $\delta$ function in the LS equation (21) will result in a nonzero $T^{z z}$, in contrast with Eq. (28). Furthermore, the $T$ matrix would have the unwanted effect that the transmitted part of an incoming wave would not be parallel to the incoming wave. The conclusion is that a regularization of the Green function was necessary, even when in the end the cutoff could be sent to infinity.

Equation (28) defines a true mode of the electromagnetic field in the presence of a single plane scatterer, in terms of a linearly polarized incoming plane wave with arbitrary angle of incidence. This is not the complete set of modes. Other modes, not corresponding to an incoming wave, will be discussed in Sec. IV B, both for a single plane and for a crystal of planes.

\section{Transmission and energy conservation}

The transmission of light through the plane can be found by choosing $z>z_{\alpha}$ in Eq. (28). The transmitted wave can be expressed in terms of the incoming wave as $\mathbf{E}_{\mathbf{k} \boldsymbol{\sigma}}\left(\mathbf{k}_{\|}, z, \omega\right)$ $=\boldsymbol{\tau}\left(\mathbf{k}_{\|}, \omega\right) \cdot \mathbf{E}_{\mathbf{k} \boldsymbol{\sigma}, 0}\left(\mathbf{k}_{\|}, z, \omega\right)$, with the transmission matrix

$$
\tau\left(\mathbf{k}_{\|}, \omega\right)=\left(\begin{array}{ccc}
\tau_{s s}\left(\mathbf{k}_{\|}, \omega\right) & 0 & 0 \\
0 & \tau_{v v}\left(\mathbf{k}_{\|}, \omega\right) & 0 \\
0 & \tau_{z v}\left(\mathbf{k}_{\|}, \omega\right) & 1
\end{array}\right),
$$

which has nonzero elements $\tau_{j j}\left(\mathbf{k}_{\|}, \omega\right)=[1-$ $\left.V(\omega) G_{0}^{j j}\left(k_{\|}, z_{\alpha}, z_{\alpha}, \omega\right)\right]^{-1} \quad$ for $j=s, v$. Furthermore, $\tau_{z v}\left(\mathbf{k}_{\|}, \omega\right)=G^{z v}\left(k_{\|}, z_{\alpha}, z_{\alpha}, \omega\right) T^{v v}\left(k_{\|}, \omega\right)$. Both for purely $s$-polarized and for purely $p$-polarized light, the transmitted electric field is a polarization-dependent scalar times the incoming electric-field vector.

Energy conservation puts a constraint (called "optical theorem") on the form that the $T$ matrix of an elastic scat- terer can take. The optical theorem for a plane that scatters scalar waves was found before [5]. Since $s$ waves map on scalar waves, the optical theorem for the ss component of the $T$ matrix can be given immediately:

$$
\operatorname{Im} T^{s s}\left(\mathbf{k}_{\|}, \omega\right)=-\frac{1}{2} \frac{\left|T^{s s}\left(\mathbf{k}_{\|}, \omega\right)\right|^{2}}{k_{z}} .
$$

The most general $T$ matrix satisfying this requirement has the form

$$
T^{s s}\left(\mathbf{k}_{\|}, \omega\right)=-\left[F_{s}^{-1}\left(\mathbf{k}_{\|}, \omega\right)-i /\left(2 k_{z}\right)\right]^{-1}
$$

where the optical potential $F_{s}\left(\mathbf{k}_{\|}, \omega\right)$ must be a real-valued function.

For reflection and transmission of $p$-polarized light, only the matrix element $T^{v v}$ is important. Again, we are interested in the form that this matrix element can take when optical energy is conserved. This is the case when the $\hat{\mathbf{z}}$ component of the Poynting vector is the same before and after the plane. An incoming $p$-polarized plane wave gives the electric field (28). With a Maxwell equation the accompanying magnetic field $\mathbf{B}$ can also be found. In SI units, and in terms of the complex fields $\mathbf{E}$ and $\mathbf{B}$, the cycle-averaged Poynting vector is equal to $\operatorname{Re}\left[\mathbf{E}^{*}(\mathbf{r}, t) \times \mathbf{B}(\mathbf{r}, t)\right] /\left(2 \mu_{0}\right)$ [37]. When a harmonic wave of frequency $\omega$ coming from $z=-\infty$ scatters off the plane, the Poynting vector is proportional to 1 $-\left(k_{z} c / \omega\right)^{2}\left|T^{v v}\right|^{2} / 4$ for $z<z_{\alpha}$. At the other side of the plane one finds $\left|1-i k_{z}(c / \omega)^{2} T^{v v} / 2\right|^{2}$. By equating the two, the optical theorem for a plane that scatters $p$-polarized light is found to be

$$
\operatorname{Im} T^{v v}\left(\mathbf{k}_{\|}, \omega\right)=\frac{-k_{z}}{2(\omega / c)^{2}}\left|T^{v v}\left(\mathbf{k}_{\|}, \omega\right)\right|^{2} .
$$

This differs from the optical theorem for $s$-polarized light. Also, the most general solution of the optical theorem is different:

$$
T^{v v}\left(\mathbf{k}_{\|}, \omega\right)=-\left[F_{p}^{-1}\left(\mathbf{k}_{\|}, \omega\right)-\frac{i k_{z}}{2(\omega / c)^{2}}\right]^{-1},
$$

where the optical potential $F_{p}\left(\mathbf{k}_{\|}, \omega\right)$ is real.

\section{E. $T$ matrix for $N$ planes}

Now that the Green function and the $T$ matrix of a single plane are known, a multiple-scattering theory can be set up. Assume that there are $N$ plane scatterers, placed at arbitrary positions. Assume them to be parallel, so that $s$ - and $p$-polarized light do not mix in the scattering process.

In the general expression (12) for the $T$ matrix of a complex dielectric in terms of its simple parts, Green functions are always sandwiched between $T$ matrices of scatterers at different positions. For unequal plane positions $z_{\alpha}$ and $z_{\beta}$, the value of $\tilde{\mathbf{G}}_{0}\left(\mathbf{k}_{\|}, z_{\beta}, z_{\alpha}, \omega\right)$ is finite and it can be taken to be equal to the unregularized $\mathbf{G}_{0}\left(\mathbf{k}_{\|}, z_{\beta}, z_{\alpha}, \omega\right)$, because different planes are at optical distances apart. Further regular- 
izations are therefore not required in order to find the $N$-plane $T$ matrix.

As shown in the Appendix, higher-order terms in the series (12) correspond to higher-order matrix multiplications of $N \times N$ matrices. The multiplication property makes that for parallel planes, the series (12) can be summed exactly. In the Appendix it is shown how the summation can be done even in the general case where all planes may have different optical properties, and are placed at arbitrary noncoinciding positions. Here we specify that all planes are identical. This gives the central result of this section, the $N$-plane $T$ matrix for scattering by vector waves:

$$
\mathbf{T}^{(N)}(\omega)=\frac{1}{(2 \pi)^{2}} \int d^{2} \mathbf{k}_{\|} \sum_{\alpha, \beta=1}^{N}\left|\mathbf{k}_{\|}, z_{\alpha}\right\rangle \mathbf{T}_{\alpha \beta}^{(N)}\left(\mathbf{k}_{\|}, \omega\right)\left\langle\mathbf{k}_{\|}, z_{\beta}\right| .
$$

Each $\alpha \beta$ component $\mathbf{T}_{\alpha \beta}^{(N)}\left(\mathbf{k}_{\|}, \omega\right)$ is a $3 \times 3$ matrix; the only two nonzero spatial components are

$$
\begin{gathered}
T_{\alpha \beta}^{s,(N)}=T^{s s}\left[\mathbf{I}_{N}-\mathcal{W}_{s} T^{s s}\right]_{\alpha \beta}^{-1}, \\
T_{\alpha \beta}^{v v,(N)}=T^{v v}\left[\mathbf{I}_{N}-\mathcal{W}_{v} T^{v v}\right]_{\alpha \beta}^{-1},
\end{gathered}
$$

Here, $\mathbf{I}_{N}$ is the $N \times N$ unit matrix. Arguments $\left(\mathbf{k}_{\|}, \omega\right)$ were temporarily dropped for readability. The $N^{2}$ matrix elements $\left(\mathcal{W}_{j}\right)_{\alpha \beta}\left(\mathbf{k}_{\|}, \omega\right)$ are defined as $\left(1-\delta_{\alpha \beta}\right) G_{0}^{j j}\left(\mathbf{k}_{\|}, z_{\alpha}, z_{\beta}, \omega\right)$, for $j=s, v$. The calculation of $\mathbf{T}^{(N)}\left(\mathbf{k}_{\|}, \omega\right)$ boils down to the inversion of an $N \times N$ matrix for the two transverse polarization directions separately.

From now on, assume that the $N$ planes are placed at regular distances from each other, with a spacing $a$ between neighbors. The necessary matrix inversions in Eq. (35) can then be performed analytically for both polarization directions. The $s$-wave case maps identically on the situation for scalar waves, for which the analytical inversion was discussed at length in Ref. [5]; for $p$ waves the inversion trick goes analogous and it will not be presented here.

A result from the analytical inversion is that $T$-matrix elements and therefore the optical properties of the $N$-plane crystal strongly depend on the Bloch wave vectors $K_{s}\left(\mathbf{k}_{\|}, \omega\right)$ and $K_{p}\left(\mathbf{k}_{\|}, \omega\right)$. For $p$-polarized light the Bloch wave vector is given by $\arccos \left(C_{p}\right) / a$, with $C_{p}=\cos \left(k_{z} a\right)+C_{p}^{\prime} \sin \left(k_{z} a\right)$; the constant $C_{p}^{\prime}$ in terms of the single-plane $T$ matrix is

$$
\frac{i k_{z}\left[k_{z}\left|T^{v v}\right|^{2}+2(\omega / c)^{2} \operatorname{Im} T^{v v}\right]+2 k_{z}(\omega / c)^{2} \operatorname{Re} T^{v v}}{k_{z}^{2}\left(\operatorname{Re} T^{v v}\right)^{2}+\left[2(\omega / c)^{2}+k_{z} \operatorname{Im} T^{v v}\right]^{2}} .
$$

In general, $C_{p}$ is a complex constant. However, if the optical theorem (32) holds, then the imaginary part of $C_{p}$ becomes identically zero, and the single-plane $T$ matrix will be of the form (33). Likewise, $K_{s}$ is defined as $\arccos \left(C_{s}\right) / a$ for a quantity $C_{s}$ that becomes real when the optical theorem, Eq. (30), for $s$-polarized light holds [5]. In those cases, the expressions for $C_{s, p}$ become rather simple,

$$
C_{s}=\cos \left(k_{z} a\right)-\left(\frac{F_{s}\left(\mathbf{k}_{\|}, \omega\right)}{2 k_{z}}\right) \sin \left(k_{z} a\right),
$$

$$
C_{p}=\cos \left(k_{z} a\right)-\left(\frac{k_{z} c^{2} F_{p}\left(\mathbf{k}_{\|}, \omega\right)}{2 \omega^{2}}\right) \sin \left(k_{z} a\right) .
$$

\section{F. A model for the optical potential}

The most general $T$ matrices (31) and (33) feature as yet unspecified optical potentials $F_{s, p}$. These should be real when energy is conserved, but for the rest they can be arbitrary functions with the frequency and the in-plane wave vector as variables.

In Ref. [5], plane scatterers were introduced as a simplified model for dielectric slabs of finite thickness $d$ and nondispersive dielectric function $\varepsilon(\omega)=\varepsilon$. The optical potential for the plane scatterer in this model is obtained via the limiting process of making the thickness $d$ of the dielectric slab smaller and increasing the polarizability $(\varepsilon-1)$, while keeping their product constant and equal to the "effective thickness" $D_{\text {eff }}$. (The quantity $D_{\text {eff }} / a$ is called the "grating strength" in Refs. $[19,20]$.) Following the same limiting procedure as in Ref. [5], we find the optical potential $F_{s, p}\left(\mathbf{k}_{\|}, \omega\right)=-V(\omega)=D_{\text {eff }}(\omega / c)^{2}$, identical for the two polarizations. Spatial dispersion and anisotropy would have shown up in the optical potentials as a $k_{\|}$and $\hat{\mathbf{k}}_{\|}$dependence, respectively. These two phenomena were neglected already as early as in the wave equation (4).

In general, $p$-polarized light differs from s-polarized light, in that the former will have a Brewster angle at which no light is reflected from a dielectric interface $\left(n_{1} \rightarrow n_{2}\right)$. The Brewster angle $\theta_{B}$ equals $\tan ^{-1}\left(n_{2} / n_{1}\right)$. In the limiting procedure for going from a finite slab-in-air to an infinitely thin plane-in-air, the dielectric contrast $\sqrt{\varepsilon} / 1$ goes to infinity and consequently the Brewster angle becomes $90^{\circ}$ in that limit. Therefore, in our limiting procedure, a plane scatterer will not have a Brewster angle at the same angle as the finite dielectric slab that one starts out with. In line with this, in Ref. [15] a single-plane reflection for $p$-polarized light was determined that is nonzero for all angles of incidence. The $p$-polarized propagating modes for a system of plane scatterers will therefore differ substantially from the corresponding modes in a slab structure. The absence of a Brewster effect was also noticed in Ref. [18] where the infinite-crystal version of the plane-scatterer model is treated.

\section{OPTICAL MODES AND OMNIDIRECTIONAL MIRRORS}

\section{A. Propagating modes}

The optical modes are the harmonic solutions of the wave equation (4). With the solution (34) of the $T$ matrix, the modes that correspond to a nonzero incoming plane wave can be given explicitly as

$$
\begin{aligned}
& \mathbf{E}_{\mathbf{k} \boldsymbol{\sigma}\left(\mathbf{k}_{\|}, z, \omega\right)}=E_{0} \boldsymbol{\sigma}_{\mathbf{k}} e^{i k_{z} z} \\
& \quad+\sum_{\alpha, \beta} \mathbf{G}_{0}\left(\mathbf{k}_{\|}, z, z_{\alpha}, \omega\right) \cdot \mathbf{T}_{\alpha \beta}^{(N)}\left(\mathbf{k}_{\|}, \omega\right) \cdot \boldsymbol{\sigma}_{\mathbf{k}} E_{0} e^{i k_{z} z \beta} .
\end{aligned}
$$



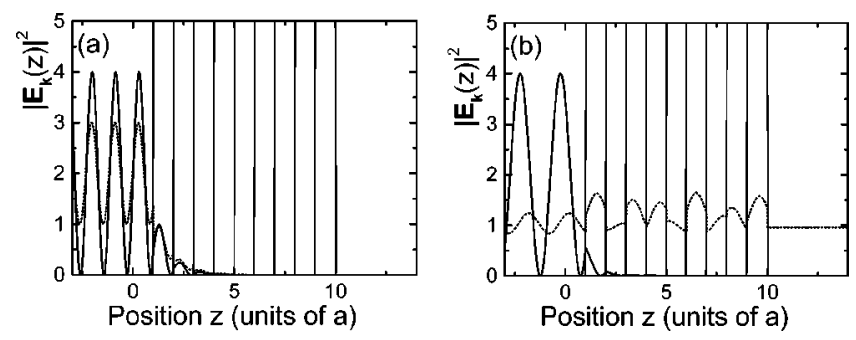

FIG. 1. Squares of absolute values of mode functions for $s$-polarized (solid lines) and $p$-polarized light (dashed lines), as a function of position. The light is scattered by a crystal of ten planes with $D_{\text {eff }}=0.46 a$, separated by a distance $a$. Both modes correspond to light incoming from the left with $a / \lambda=0.5$. Panel (a), $\theta_{\text {in }}=30^{\circ}$; panel (b), $\theta_{\text {in }}=60^{\circ}$.

These propagating (or radiative) modes are labeled by the incoming wave vector $\mathbf{k}$ and polarization $\boldsymbol{\sigma}_{\mathbf{k}}$. The sine of the angle of incidence (with respect to a vector normal to the planes) is equal to $k_{\|} c / \omega$. The amplitudes of the $s$-polarized modes [with $\left.\boldsymbol{\sigma}_{\mathbf{k}}=(1,0,0)\right]$ are identical to the corresponding amplitudes for scalar waves; the $p$-polarized modes $\left[\boldsymbol{\sigma}_{\mathbf{k}}\right.$ $\left.=\left(0, k_{z} / k,-k_{\|} / k\right)\right]$ have no scalar analogs.

For light with wave vector and frequency such that $C_{s, p}$ $>1$ [see Eq. (37)], the Bloch wave vector is purely imaginary for the elastic scatterers that we consider. Similarly, for $C_{s, p}<-1$, the Bloch wave vector equals $\pi$ plus an imaginary number. In both situations the light will feel a stop band, meaning that it will be $100 \%$ reflected when falling on a semi-infinite system of planes. Otherwise, when -1 $<C_{s, p}<1$, the Bloch wave vector is real and light can propagate inside the crystal. More will be said about the Bloch wave vectors later in this section.

Some plots of mode profiles will now be presented. Assume that light comes in from the left. For perpendicularly incident light, there is no difference between $s$ and $p$ polarization. In Fig. 1 the mode profiles (or squared absolute values of mode functions) for $s$ - and $p$-polarized light inside a ten-plane crystal are compared both for an incoming angle of $30^{\circ}$ and for $60^{\circ}$. Figure 1(a) shows that at an angle of $30^{\circ}$ the mode profiles corresponding to both polarizations do not differ much yet. Both modes decay rapidly inside the crystal structure and are reflected (almost) completely. The Bloch wave vectors are complex for both polarizations. Only for the $s$ wave the polarization directions of the incoming and the reflected wave are equal, so that the amplitude of its mode profile at the left side of the crystal is four times the amplitude of the incoming electric field.

The situation is different at an incoming angle of $60^{\circ}$, as shown in Fig. 1(b). The mode profile of the s-polarized light again rapidly decays inside the crystal (and the corresponding Bloch wave vector again has an imaginary part), whereas the $p$-polarized light can propagate inside the crystal and is transmitted almost completely (and the Bloch wave vector is real). For this frequency and incoming angle, the crystal is a good polarization filter.

The mode profiles in Figs. 1(a,b) of the $s$-polarized waves are continuous whereas $p$-polarized waves are discontinuous at the positions of the planes. This reflects the boundary con-
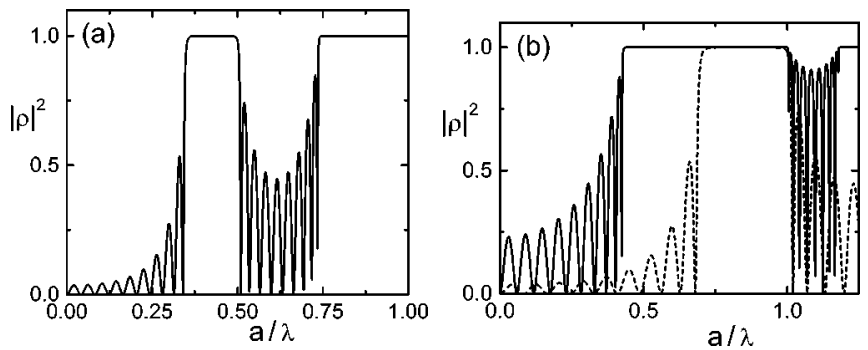

FIG. 2. Reflection off a ten-plane crystal, as a function of $a / \lambda$, for $s$-polarized light (solid lines) and $p$-polarized light (dashed lines). Angles of incidence are $0^{\circ}$ in (a) and $60^{\circ}$ in (b). The planes have effective thickness $D_{\text {eff }}=0.46 a$ and they are separated by a distance $a$. In (a), the graphs for $s$ and $p$ polarization overlap.

ditions: the tangential components of the electric fields must be continuous and the normal components must show a jump at a dielectric interface. The electric field of $s$-polarized light only has a tangential component, while $p$-polarized light consists of both tangential and normal components. This explains the differences in the mode profiles for $s$ and $p$ waves. Notice that in our Green-function formalism, boundary conditions are automatically satisfied, whereas in related work based on transfer-matrix methods, boundary conditions must be considered explicitly $[15,16,20]$.

Reflection as a function of frequency by the ten-plane Bragg mirror is plotted in Fig. 2 for both polarization directions. The reflection $|\rho|^{2}$ equals $\left(1-|\tau|^{2}\right)$, with $|\tau|^{2}$ the (relative) transmitted light intensity. For light incident perpendicularly to the planes, both transverse polarization vectors are equivalent and accordingly in Fig. 2(a), the graphs for $s$ - and $p$-polarized light overlap. Differences between the two polarizations do appear for non-normal incidence. In Fig. 2(b) the angle of incidence is $60^{\circ}$. The red edges of the stop bands for $s$-polarized light move to slightly higher frequencies and the widths of the stop bands become larger. For $p$-polarized light the red edges of the stop bands shift to the blue much faster, and the faster so for larger angles of incidence.

For scalar waves, a crystal of plane scatterers can be an omnidirectional mirror [5], which means that waves experience a stop band for all angles of incidence. For vector waves, the crystal will only be an omnidirectional mirror if there are frequency intervals in which the crystal is an omnidirectional mirror both for $s$ and $p$ waves.

As stated earlier, it is the Bloch wave vectors $K$ that distinguish between light that can propagate inside the crystal (real $K$ ) and light that feels a stop band (when $K$ is an imaginary number or $\pi$ plus an imaginary number). In our formalism, the Bloch wave vectors are the arccosines of the constants $C_{s}$ and $C_{p}$ given in Eq. (37). As is shown in detail in Ref. [5], these Bloch wave vectors show up in expressions for the $N$-plane $T$ matrix $T^{(N)}$. It must be said that in the present $T$ matrix formalism it is not obvious simply by looking at the equations that a stop band occurs whenever the Bloch wave vector has a nonzero imaginary part. Nevertheless, we conclude from our numerical calculations that the relation does exist. To give an example, of the four modes in the Figs. 1(a,b), only the $p$-polarized light incoming at $60^{\circ}$ 


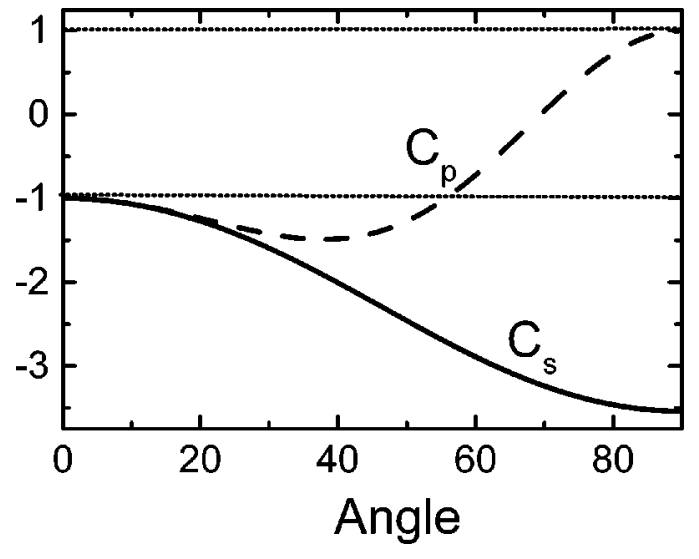

FIG. 3. Constants $C_{s}$ and $C_{p}$ as a function of angle of the incoming light, for the parameters $a / \lambda=0.5$ and $D_{\text {eff }}=0.46 a$. Regions where $-1 \leqslant C \leqslant 1$ correspond to propagating waves inside the crystal.

has a corresponding real Bloch wave vector. It is an interesting fact that the Bloch wave vector, the role of which is obvious in infinite crystals, also plays an important role in finite periodic structures. This was already noticed before in the context of transfer-matrix methods [27-29]; here we see the importance of the Bloch wave vector for finite periodic structures in a $T$-matrix formalism.

For light of a frequency corresponding to $a / \lambda=0.5$ and planes with $D_{\text {eff }}=0.46 a$, the $s$ waves are reflected omnidirectionally [5]. In Fig. 3 we plot both constants $C_{s}$ and $C_{p}$ for this frequency, as a function of angle of incidence. Unlike for $s$ waves, for $p$ waves there are incident angles larger than the critical angle $\theta_{c} \gtrsim 55^{\circ}$ for which the values of $C_{p}$ are between -1 and 1 . Light incident with these large angles can propagate inside the crystal and therefore the crystal is not an omnidirectional mirror for this frequency. Actually, this information could already be read off from the mode profile of $p$-polarized light incident at $60^{\circ}$ in Fig. 1(b). The conclusion holds more generally: for larger $D_{\text {eff }}$ the critical angle $\theta_{c}$ increases, but it can be shown by expanding Eq. (37b) around $\theta_{\text {in }}=90^{\circ}$ that for every finite $D_{\text {eff }} / a$ and $a / \lambda$ there always is a finite interval of angles corresponding to propagating $p$-polarized light. In conclusion, crystals of identical and equidistant plane scatterers can reflect vector waves in almost all (but not in all) directions.

\section{B. Guided modes}

Besides propagating modes there can also be bound modes that do not correspond to incoming light (see Sec. II). Bound modes can be found by solving the LS equation in the absence of an incoming field. In crystals of plane scatterers, bound modes are guided modes. They have imaginary wave vectors in the $z$ direction and they decay exponentially away from the planes. Their in-plane wave vectors $k_{\|}$are larger than $\omega / c$. With each mode, be it of the propagating or guided type, a nonzero local density of states is associated. In the following, guided modes will be searched by looking for nonzero densities of states. (This is a method alternative to the one used in Ref. [5] where guided modes of scalar waves were identified.)
For vector waves, the local optical density of states $N(\mathbf{r}, \omega)$ is defined by [7]

$$
-\left[(2 \omega) /\left(\pi c^{2}\right)\right] \operatorname{Im} \operatorname{Tr} \mathbf{G}(\mathbf{r}, \mathbf{r}, \omega),
$$

so it is a scalar proportional to the trace over the imaginary part of the Green tensor $\mathbf{G}(\mathbf{r}, \mathbf{r}, \omega)$. In planar geometries the latter can best be found as an integral over the Green tensor in the plane representation:

$$
\mathbf{G}(\mathbf{r}, \mathbf{r}, \omega)=\frac{1}{(2 \pi)^{2}} \int d^{2} \mathbf{k}_{\|} \mathbf{G}\left(\mathbf{k}_{\|}, z, z, \omega\right)
$$

The local density of states can only be nonzero if the imaginary part of the integrand in (40) is nonzero. A guided mode manifests itself when diagonal elements of this integrand $\mathbf{G}\left(\mathbf{k}_{\|}, z, z, \omega\right)$ have nonzero imaginary parts for a certain $k_{\|}$ $>\omega / c$. For the crystals of plane scatterers the Green tensor directly follows from the Dyson-Schwinger equation:

$$
\begin{aligned}
\mathbf{G}\left(\mathbf{k}_{\|}, z, z^{\prime}, \omega\right) & \\
= & \mathbf{G}_{0}\left(\mathbf{k}_{\|}, z, z^{\prime}, \omega\right) \\
& +\sum_{\alpha, \beta=1}^{N} \mathbf{G}_{0}\left(\mathbf{k}_{\|}, z, z_{\alpha}, \omega\right) \cdot \mathbf{T}_{\alpha \beta}^{(N)}\left(\mathbf{k}_{\|}, \omega\right) \cdot \mathbf{G}_{0}\left(\mathbf{k}_{\|}, z_{\beta}, z^{\prime}, \omega\right) .
\end{aligned}
$$

All three diagonal components of $\mathbf{G}_{0}\left(\mathbf{k}_{\|}, z, z^{\prime}, \omega\right)$ become real quantities for $k_{\|}>\omega / c$, and indeed there are no guided modes in free space. On the other hand, the off-diagonal elements $G_{0}^{v z}=G_{0}^{z v}$ become purely imaginary when $k_{\|}$ $>\omega / c$ and the latter elements do show up in the diagonal elements of $\mathbf{G}$. However, since they always show up in paired products, for example, in the term $G_{0}^{z v} T_{\alpha \beta}^{(N), v v} G_{0}^{v z}$, they also give a real contribution to diagonal elements of $\mathbf{G}$. The $T$-matrix elements are also real when $k_{\|}>\omega / c$, except when the matrix has a pole. Therefore, all guided modes must correspond to poles of the $s$ or $p$ components of the $N$-plane $T$ matrix $\mathbf{T}^{(N)}$ [see Eq. (35)].

First, the guided modes of a single plane will be determined. There can be a guided mode when either $T^{s s}$ or $T^{v v}$ in Eq. (28) has a pole. Now $T^{s s}$ has a pole when 1 $-V(\omega) G_{0}^{s s}\left(\mathbf{k}_{\|}, z_{\alpha}, z_{\alpha}, \omega\right)$ vanishes. Using the same model for the optical potential as in Sec. III F, we find the dispersion relation $\kappa_{1}^{(1)}=D_{\text {eff }}(\omega / c)^{2} / 2$ for one and only one guided mode corresponding to $s$-polarized light. Here, $\kappa$ is the positive square root $\left[k_{\|}^{2}-(\omega / c)^{2}\right]^{1 / 2}$ for $k_{\|}>\omega / c$. A single-plane guided mode with this dispersion relation was also found in Ref. [15], and in Ref. [5] for scalar waves.

A pole of $T^{v v}$ occurs when $1-V(\omega) G_{0}^{v v}\left(\mathbf{k}_{\|}, z_{\alpha}, z_{\alpha}, \omega\right)$ vanishes, which is equivalent to the requirement that $D_{\text {eff }} \kappa / 2$ equals -1 . Now, in principle, $D_{\text {eff }}$ could be negative when modeling a slab of negative dielectric function as a plane scatterer. However, in the physical situations that we are interested in, the effective thickness is real and positive (see Sec. III F). Therefore, there is no guided mode corresponding to $p$-polarized light for a single-plane scatterer. This result 
was also derived in Ref. [15], where only the cases of a single plane and infinitely many planes were considered.

At this point it is worthwhile to compare the guided modes of a dielectric slab (thickness $d$, dielectric constant $\varepsilon$ ) in air with the guided modes of an infinitely thin plane with effective thickness $D_{\text {eff }}=(\varepsilon-1) d$. For the slab, the number $M$ of guided modes is the same for both polarizations in the special case considered here, and equal to [38]

$$
M_{s, p}=1+[2 d \sqrt{\varepsilon-1} / \lambda] .
$$

Here, $[X]$ stands for the largest integer smaller than or equal to $X$. In the large-wavelength limit there is a single guided mode for each polarization direction. The second guided mode appears when $a / \lambda=a /\left(2 \sqrt{d D_{\text {eff }}}\right)$. For example, for $d$ $=0.1 a$ and $D_{\text {eff }}=0.46 a$, a second guided mode exists when $a / \lambda>2.3$. We are interested in frequencies around $a / \lambda$ $=0.5$, where the first stop band for normally incident light occurs (see Fig. 2). For these frequencies, both the plane scatterer and the dielectric slab have a single s-polarized guided mode; the slab has a single $p$-polarized guided mode, whereas the plane scatterer has no such guided mode at all.

Now we determine the guided modes of a finite crystal of $N$ parallel and equidistant planes, using the same Greenfunction method as for the single plane. First look for the poles of the component $T_{\alpha \beta}^{s s,(N)}\left(\mathbf{k}_{\|}, \omega\right)$. This is easy, because this component is identical to the $N$-plane $T$ matrix $T^{(N)}$ for scalar waves, for which it was found that there are at most $N$ guided modes in a crystal of $N$ planes [5]. For an infinite number of planes, the guided modes form a band, as was found in Refs. $[15,19]$. Now look for guided modes corresponding to $p$-polarized light. The poles of the component $T_{\alpha \beta}^{v v,(N)}\left(\mathbf{k}_{\|}, \omega\right)$ occur when the $\operatorname{determinant} \operatorname{det}\left[\left(\mathbf{T}^{v v,(N)}\right)^{-1}\right]$ is equal to zero. An expression for this determinant can be found just as was done for scalar waves in Ref. [5]. The result is that for $p$-polarized light there are guided modes if the following equation has nontrivial solutions $\omega(\kappa)$ :

$$
\sin \left[(N+1) K_{p} a\right]+\left[\frac{\kappa F-2(\omega / c)^{2}}{2(\omega / c)^{2}}\right] e^{-\kappa a} \sin \left(N K_{p} a\right)=0 .
$$

The Bloch wave vector $K_{p}$ is still defined as $a^{-1}$ times the arccosine of $C_{p}$, which reads $C_{p}=\cosh (\kappa a)$ $+\left[\left(\kappa c^{2} F\right) /\left(2 \omega^{2}\right)\right] \sinh (\kappa a)$ in terms of $\kappa$.

Equation (43) should lead to the dispersion relations $\omega(\kappa)$ for the guided modes, if they exist. When increasing the frequency, new guided modes would appear that at first are only just captured by the structure so that $\kappa=0^{+}$. It is therefore convenient to count the guided modes in the small- $\kappa$ limit. Let the constant $\chi$ be defined as $a \sqrt{1+F c^{2} /\left(a \omega^{2}\right)}$. Then $C_{p}$ can be written up to second order in $\kappa$ as

$$
C_{p}=1+(\chi \kappa)^{2} / 2+o\left(\kappa^{3}\right)=\cosh (\chi \kappa)+o\left(\kappa^{3}\right) .
$$

To the same order in $\kappa$, the Bloch wave vector $K_{p}$ becomes equal to $i \chi \kappa / a$. Therefore, solutions of Eq. (43) will only exist when $\sinh [(N+1) \chi \kappa]$ equals $\sinh (N \chi \kappa)$, or equivalently when $\chi \equiv 0$. Since $F$ is taken to be $D_{\text {eff }}(\omega / c)^{2}$, there are guided modes for $p$-polarized light whenever $1+D_{\text {eff }} / a$ $=0$. So in finite crystals of $N$ planes with a positive effective thickness, there are no guided modes corresponding to $p$-polarized light. This is in agreement with the result just obtained for the single plane, and with the results in Refs. $[15,20]$ for infinite crystals.

In conclusion, there are at most $N$ guided modes in the finite crystal of plane scatterers, all modes corresponding to $s$-polarized light. The comparison of the single plane and the single slab indicates that $s$ waves in plane scatterers are a good model for $s$ waves in slabs, at least for frequencies around the first stop band. For the $p$-polarized guided modes, the conclusion must be that in the finite slab structures there are guided modes which have no analogs in the crystal of plane scatterers.

\section{SPONTANEOUS EMISSION}

\section{A. Application to layered dielectrics}

In free space, the spontaneous-emission rate $\Gamma_{0}$ of an atom with dipole moment $\boldsymbol{\mu}$ and transition frequency $\Omega$ equals $\mu^{2} \Omega^{3} /\left(3 \pi \hbar \varepsilon_{0} c^{3}\right)$. When embedded in an inhomogeneous dielectric, the rate $\Gamma$ will in general be different, as can be found with Fermi's golden rule [39],

$$
\Gamma(\boldsymbol{\mu}, \mathbf{R}, \Omega)=\pi \sum_{l} \frac{\omega_{l}}{\hbar \varepsilon_{0}}\left|\boldsymbol{\mu} \cdot \mathbf{E}_{l}(\mathbf{R})\right|^{2} \delta\left(\omega_{l}-\Omega\right) .
$$

$\mathbf{E}_{l}$ are the normal-mode solutions with eigenfrequencies $\omega_{l}$ of the wave equation (4). The spontaneous-emission rate can alternatively be expressed in terms of the Green function of the medium

$$
\Gamma(\boldsymbol{\mu}, \mathbf{R}, \Omega)=\frac{-2 \Omega^{2}}{\hbar \varepsilon_{0} c^{2}} \operatorname{Im}[\boldsymbol{\mu} \cdot \mathbf{G}(\mathbf{R}, \mathbf{R}, \Omega) \cdot \boldsymbol{\mu}] .
$$

(See Ref. [40] for early derivations of this relation; in Ref. [41] a modern derivation is given for inhomogeneous and absorbing dielectric media.) In Eq. (46), G is the classical dyadic Green function of the electric-field wave equation (4). For homogeneous dielectrics, it is known that the total Green function is the sum of a transverse part that describes radiative decay and a longitudinal part describing nonradiative decay [42]. Here, Eqs. (45) and (46) are equivalent, because nonradiative decay is absent for dielectrics with real dielectric functions.

Layered dielectrics (not necessarily plane scatterers) are translation invariant in two directions, which can be chosen to be the $(\hat{\boldsymbol{x}}, \hat{\boldsymbol{y}})$ directions. Spontaneous-emission rates will only depend on the $z$ coordinate of the atomic position $\mathbf{R}$ $=(x, y, z)$. It is then easiest to first calculate the Green function in the plane representation $\mathbf{G}\left(\mathbf{k}_{\|}, z, z, \Omega\right)$. This Green function must be Fourier transformed back to real space as in Eq. (40) in order to find the local Green function of Eq. (46) that determines spontaneous-emission rates.

A slight complication in doing the integration (40) is that the plane representation for $\mathbf{G}\left(\mathbf{k}_{\|}, z, z, \Omega\right)$ is corotating with the incoming wave vector $\mathbf{k}_{\|}$, a variable that must now be 
integrated over. A fixed basis $\{\hat{\mathbf{x}}, \hat{\mathbf{y}}, \hat{\mathbf{z}}\}$ is needed instead and it is chosen such that the atomic dipole becomes $\left(\mu_{x}, 0, \mu_{z}\right)$ in the new representation. Write the two-dimensional integral $\int d^{2} \mathbf{k}_{\|}$in polar coordinates as $\int_{0}^{\infty} d k_{\|} k_{\|} \int_{0}^{2 \pi} d \hat{\mathbf{k}}_{\|}$. After doing the angular integration, only diagonal elements of the dyadic Green function survive.

The total spontaneous-emission rate is the sum of two contributions, the perpendicular and the parallel decay rate,

$$
\begin{gathered}
\Gamma_{z}(z, \Omega)=-\frac{3 c \Gamma_{0} \mu_{z}^{2}}{\Omega \mu^{2}} \operatorname{Im} \int_{0}^{\infty} d k_{\|} k_{\|} G^{z z} \\
\Gamma_{x}(z, \Omega)=-\frac{3 c \Gamma_{0} \mu_{x}^{2}}{\Omega \mu^{2}} \operatorname{Im} \int_{0}^{\infty} d k_{\|} k_{\|} \frac{G^{s s}+G^{v v}}{2} .
\end{gathered}
$$

[Green-function arguments $\left(k_{\|}, z, z, \Omega\right)$ were again dropped.] The parallel decay rate has a contribution both from $s$ - and $p$-polarized light (through $G^{s s}$ and $G^{v v}$, respectively) whereas the perpendicular decay rate only has a $p$-polarized decay channel (through $G^{z z}$ ). Notice that the (real) $\delta$-function term in $G_{0}^{z z}$ does not play a role in the emission rates. The spontaneous-emission rates in Eqs. (47a) and (47b) are integrals over all possible lengths of the in-plane wave vector. Both rates can be subdivided into a propagating-mode (or radiative-mode) rate corresponding to the integration of $k_{\|}$from 0 to $\Omega / c$, and a guided-mode rate which is the integral from $\Omega / c$ to $\infty$.

\section{B. Spontaneous emission near plane scatterers}

The general expressions obtained in Sec. V A for spontaneous emission in layered structures will now be applied to crystals of plane scatterers. Combine the general expressions (47) for spontaneous-emission rates in layered dielectrics with the Green functions in the plane representation that were determined in Eq. (41) for a crystal of plane scatterers. Because of the absence of $p$-polarized guided modes, the parallel decay rate near plane scatterers can be subdivided into three (instead of four) parts: an $s$-polarized radiativemode rate $(s r)$, a $p$-polarized radiative-mode rate $(p r)$, and an $s$-polarized guided-mode rate $(s \mathrm{~g})$. Again, because of the absence of $p$-polarized guided modes, the perpendicular decay rate $\Gamma_{z}$ is purely radiative. Here is a list of the nonzero partial decay rates:

$$
\begin{aligned}
& \Gamma_{x}^{s \mathrm{r}}(z, \Omega)=-\frac{3 c \Gamma_{0}}{2 \Omega}\left(\frac{\mu_{x}}{\mu}\right)^{2} \operatorname{Im} \int_{0}^{\Omega / c} d k_{\|} k_{\|} G^{s s} \\
& \Gamma_{x}^{p \mathrm{r}}(z, \Omega)=-\frac{3 c \Gamma_{0}}{2 \Omega}\left(\frac{\mu_{x}}{\mu}\right)^{2} \operatorname{Im} \int_{0}^{\Omega / c} d k_{\|} k_{\|} G^{v v}, \\
& \Gamma_{x}^{s \mathrm{~g}}(z, \Omega)=-\frac{3 c \Gamma_{0}}{2 \Omega}\left(\frac{\mu_{x}}{\mu}\right)^{2} \operatorname{Im} \int_{\Omega / c}^{\infty} d k_{\|} k_{\|} G^{s s} \\
& \Gamma_{z}(z, \Omega)=-\frac{3 c \Gamma_{0}}{\Omega}\left(\frac{\mu_{z}}{\mu}\right)^{2} \operatorname{Im} \int_{0}^{\Omega / c} d k_{\|} k_{\|} G^{z z}
\end{aligned}
$$
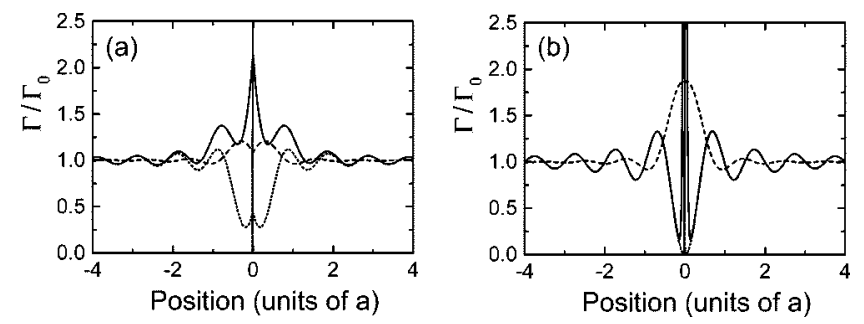

FIG. 4. Spontaneous-emission rates of dipoles near a single partially transmitting plane scatterer, relative to $\Gamma_{0}$. The wavelength is chosen such that $a / \lambda=0.5$. Solid lines correspond to total spontaneous-emission rates $\Gamma_{x}$ for dipoles parallel to the plane, dotted lines are radiative contributions to $\Gamma_{x}$, and the dashed lines denote $\Gamma_{z}$. In (a), the effective thickness $D_{\text {eff }}$ of the plane equals $0.46 a$ and in (b) $D_{\text {eff }}=10 a$.

To be precise, in Eq. (48d) it was used that the tensor element $G^{z z}$ has a vanishing imaginary part (leading to a vanishing contribution to the density of states) for $k_{\|}>\Omega / c$; for the same reason, there is no guided-mode rate analogous to Eq. (48c) corresponding to $G^{v v}$. These properties were found in Sec. IV B.

With all partial emission rates spelled out now, we first study spontaneous-emission rates near a single plane, for which the Green function in the plane representation (41) features the single-plane $T$ matrix of Eq. (28). In Fig. 4(a), spontaneous-emission rates as a function of position are plotted for $D_{\text {eff }}=0.46 a$. For both orientations of the dipole, far away from the plane the rate approaches the free-space value. Close to the plane, $\Gamma_{x}$ is larger than $\Gamma_{0}$, but it consists of a rate into propagating modes that is less than $\Gamma_{0}$, and a guided-mode rate. Close to the plane, $\Gamma_{z}$ is larger than $\Gamma_{0}$, but the maximal values of (the purely radiative) $\Gamma_{z}$ occur somewhat away from the plane.

The contribution of radiative and guided $s$ waves for an atom with $\mu=\mu_{x}$ is the same as for scalar waves with "scalar dipole moment" $\mu$, but since the total decay rate $\Gamma_{0}$ for vector waves is larger than for scalar waves, the relative contributions of $s$ waves to $\Gamma / \Gamma_{0}$ are smaller for vector waves (by a factor 3/4).

In Fig. 4(b), the same rates are plotted, this time for a plane with $D_{\text {eff }}=10 a$ that reflects light almost ideally: near the plane, $\Gamma_{z}$ is almost twice $\Gamma_{0}$. The maximum values of $\Gamma_{z}$ still occur away from the plane, although this has become invisible in Fig. 4(b). The propagating-mode part of $\Gamma_{x}$ has decreased and is practically zero on the plane. The partial emission rate into the guided mode has a much larger (but finite, not shown) amplitude near the plane. The other prominent difference in the two figures is that the "spike" in the emission rates due to the guided modes has become much narrower. Indeed, from Eqs. (39)-(41), it follows that the guided-mode rate decays exponentially away from the plane like $\exp \left(-2 \kappa_{1}^{(1)}|z|\right)$. It follows from the dispersion relation for $\kappa_{1}^{(1)}$ that was obtained in Sec. IV B, that an increase in $a / \lambda$ or in $D_{\text {eff }}$ will give narrower spikes.

In the limit that the atomic position $z$ becomes equal to the plane position $z_{\alpha}=0$, the spontaneous-emission rates can be calculated analytically for both dipole orientations. For a dipole perpendicular to the planes, 


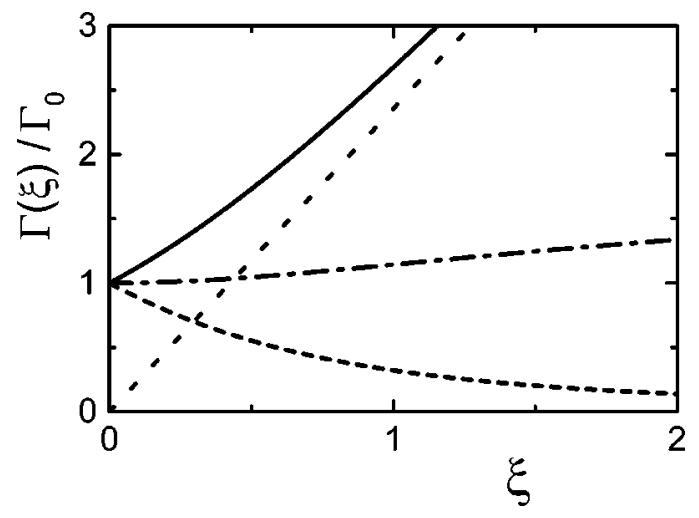

FIG. 5. Spontaneous-emission rates of dipoles at the position of the single-plane scatterer, as a function of the dimensionless parameter $\xi=\pi D_{\text {eff }} / \lambda$. The rate $\Gamma_{x}$ (solid line) is the sum of a rate into radiative (dashed line) and into guided modes (dotted line). The purely radiative-mode rate $\Gamma_{z}$ is the dash-dotted line.

$$
\Gamma_{z}\left(z_{\alpha}, \Omega\right)=\Gamma_{0}\left(2+\frac{3}{2 \xi^{2}}\right)-\frac{3}{2} \Gamma_{0}\left(1+\frac{1}{\xi^{2}}\right) \frac{\arctan (\xi)}{\xi}
$$

where the dimensionless parameter $\xi$ is defined as $\pi D_{\text {eff }} / \lambda$. Similarly, for a dipole parallel to the plane, the three partial contributions to the decay rate can also be expressed in terms of the parameter $\xi$ alone:

$$
\begin{gathered}
\Gamma_{x}^{s r}\left(z_{\alpha}, \Omega\right)=\frac{3}{4} \Gamma_{0}[1-\xi \arctan (1 / \xi)], \\
\Gamma_{x}^{p r}\left(z_{\alpha}, \Omega\right)=\frac{3}{4 \xi^{2}} \Gamma_{0}\left[1-\frac{\arctan (\xi)}{\xi}\right], \\
\Gamma_{x}^{s g}\left(z_{\alpha}, \Omega\right)=\frac{3 \pi \xi}{4} \Gamma_{0} .
\end{gathered}
$$

In Fig. 5 the relative rates are plotted as a function of $\xi$. The results can be checked in two limiting cases: if $D_{\text {eff }}=0$ there is no plane and then indeed both $\Gamma_{z}$ and $\Gamma_{x}$ are equal to the free-space value $\Gamma_{0}$. The other limit is that of a perfect mirror, when $D_{\text {eff }}$ (and consequently $\xi$ ) is sent to infinity. This limit is not visible in the figure, but the limiting values are $\Gamma_{z} / \Gamma_{0}=2$ and $\Gamma_{x} / \Gamma_{0}=0$. These values indeed agree with the well-known emission rates for atoms near perfect mirrors [21-23]. Emission rates into guided modes vanish in the perfect-mirror limit; at $z=z_{\alpha}$ this is only the case by sending $D_{\text {eff }}$ to infinity before putting $z$ equal to $z_{\alpha}=0$. This completes the discussion of emission rates near a single plane.

Now consider emission rates inside and near a crystal of a number $N$ of plane scatterers. Results will be presented for $N=10$. In Figs. 6(a)-6(d), orientation-dependent spontaneous-emission rates are plotted for several frequencies. For clarity in the pictures, the positions of the planes at $a, 2 a, \ldots, 10 a$ are not shown as vertical lines this time. The most striking difference between $\Gamma_{x}$ and $\Gamma_{z}$ is that $\Gamma_{x}$ becomes very spiky near the planes, because only parallel di-
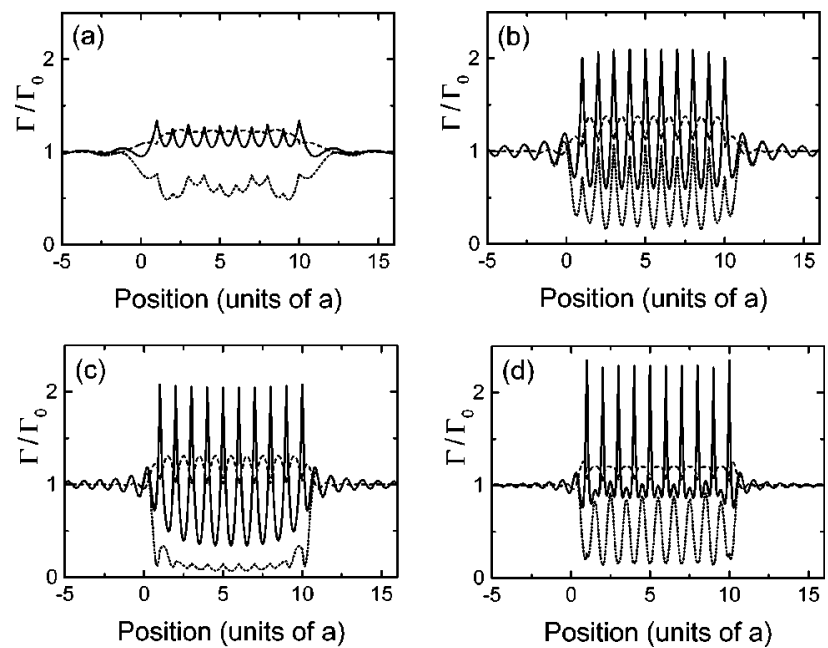

FIG. 6. Spontaneous-emission rates $\Gamma_{x}$ (solid line) and $\Gamma_{z}$ (dashed) near a ten-plane crystal. For all planes, $D_{\text {eff }}=0.46 a$. The dotted line is the radiative part of $\Gamma_{x}$. The parts (a)-(d) correspond to four frequencies: (a) $a / \lambda=0.2$, (b) $a / \lambda=0.4$, (c) $a / \lambda=0.5$, and (d) $a / \lambda=0.6$.

poles can couple to the $s$-polarized guided modes (and because $p$-polarized guided modes are absent in our model).

As the frequency increases when going from Fig. 6(a) to Fig. $6(d), \Gamma_{x}$ becomes more spiky, because the partial emission rate into the guided modes (the difference between the solid and the dotted lines in Fig. 6) becomes more concentrated near the planes. The term "concentration" is appropriate here, because the maximum amplitudes near the planes are higher for narrower spikes. The same effect was observed for the single plane in Figs. 4(a,b), where the frequency is kept constant and $D_{\text {eff }}$ is increased instead.

The purely radiative-mode rate $\Gamma_{z}$ on average increases due to the presence of the planes, whereas the radiative part of $\Gamma_{x}$ on average decreases. The same behavior occurs near a single plane in Fig. 4 and for a perfect mirror.

Figure 1 showed that the optical modes of $p$-polarized light have discontinuities at the plane positions. There are also discontinuities in the spontaneous-emission rates (not for a single plane, for symmetry reasons), but these are too small to be visible in Fig. 6. It can be understood that they are small from the fact that the discontinuities per mode are averaged in the emission rate.

The dotted lines in Fig. 6 are the radiative parts of $\Gamma_{x}$. These are similar to the radiative-mode rates for scalar waves [5], but not identical, since in $\Gamma_{x}$ not only $s$-polarized but also $p$-polarized light contributes, according to Eq. (47b). In particular, far away from the planes, the emission rate of dipoles parallel to the planes consists of $75 \% \mathrm{~s}$-polarized and $25 \% p$-polarized light. [To be sure, light emitted by perpendicular dipoles is $100 \% p$ polarized for all layered dielectrics, see Eq. (47a).]

For "large enough" photonic crystals, one expects inner unit cells to have optical properties similar to unit cells in the infinite crystal. Are the ten-plane crystals large enough? This depends on the properties of a single plane. In two extreme cases, the crystal size does not matter. When the individual 
planes do not reflect any light in any direction $\left(D_{\text {eff }} / a\right.$ $=0)$, or when they reflect all light $\left(D_{\text {eff }} / a=\infty\right)$, one finds the same emission rates in finite and in infinite crystals, because for $D_{\text {eff }} / a=0$ we have the free-space case and for $D_{\text {eff }} / a=\infty$ all unit cells are optically disconnected. Only in intermediate cases $\left(0<D_{\text {eff }} / a<\infty\right)$ can finite photonic crystals have an appreciable and unit-cell dependent influence on spontaneous-emission rates.

In the intermediate cases (we assumed $D_{\text {eff }} / a=0.46$ ), the single-plane reflection also depends on the frequency of the light. The planes reflect light better at higher frequencies, because material dispersion was neglected. It appears that for the lowest frequency considered in Fig. $6, a / \lambda=0.2$ in (a), the emission rates in inner unit cells already are influenced considerably by the crystal. The rates vary in neighboring inner unit cells, which indicates that the rates have not yet converged to the infinite-crystal values. For higher frequencies, say $a / \lambda=0.6$, individual planes reflect light much better. In the corresponding Fig. 6(d), all inner unit cells look alike and emission rates have converged.

Now consider a parallel dipole at a fixed position, very close to a plane in an inner unit cell. From Fig. 6, one can also appreciate how the guided modes will influence the (frequency-dependent) emission rate of a dipole very close to a plane. For frequencies $a / \lambda=0.4$ and higher, Figs. 6(b)$6(d)$ show that emission into propagating modes is negligible compared to emission into guided modes. The dipole falls inside the guided-mode spike near the plane. As the frequency increases, the maximum amplitude of the spike increases. With the dipole still well inside the spike, the emission rate of the dipole will increase as well. When increasing the frequency further, the spike becomes so narrow that the dipole ends up in one of the wings of the spike, until the dipole finds itself completely outside it. This will cause the emission rates into guided modes to drop at higher frequencies. The combined effect is a peak in the frequencydependent emission rates. Indeed, in Ref. [19] the dipole emission rate (or more precisely, its $s$-wave component) for infinite crystals as a function of frequency shows a pronounced peak for dipole positions $z$ near a plane (see Fig. 3 in Ref. [19]). We can unambiguously attribute this peak to emission into the guided modes.

We can also understand how an emission peak will depend on $D_{\text {eff }}$ and on the distance to the plane. We have seen that spikes are narrower, with higher amplitudes, for larger $D_{\text {eff }}$ and for higher frequencies. When assuming larger $D_{\text {eff }}$, a dipole at fixed distance will feel a guided-mode enhanced emission rate for lower frequencies. However, the dipole will also at lower frequencies begin to fall outside the range of the spike. This explains why for fixed dipole position and increased $D_{\text {eff }}$, the emission peak has a larger amplitude and attains its maximum at a lower frequency, precisely as seen in Fig. 3 in Ref. [19]. Similar reasoning suggests that for a parallel dipole a bit further away from a plane but still close to it (while keeping $D_{\text {eff }}$ fixed), an emission peak will occur at lower frequencies, with lower amplitude.

What can be appreciated best in Fig. 6(a) is that for parallel dipoles the total emission rate converges faster than the radiative and guided-mode partial rates separately. This can be related to a kind of mode competition between $s$-polarized propagating and guided modes that we also found for scalar waves [5]. On the other hand, for perpendicular dipoles all emission is into radiative modes, so mode competition is absent there and convergence sets in earlier.

For scalar waves the ten-plane structure could act as an omnidirectional mirror, whereas in Sec. IV A it was found that it is not an omnidirectional mirror for vector waves. Correspondingly, the radiative-mode LDOS for scalar waves at $a / \lambda=0.5$ dropped down to (almost) zero inside the tenplane omnidirectional mirror, whereas the emission rates in Fig. 6(c) show that the radiative LDOS for vector waves stays nonzero inside the crystal. In the inner unit cells, dipoles parallel to the planes emit predominantly guided light. The small amount of light that leaves the structure is strongly $p$ polarized. This is the case around $a / \lambda=0.5$ only, where $s$-polarized light is omnidirectionally reflected. Such strongly polarized emission is not a peculiarity of the plane-scatterer model, because it will also occur for a real Bragg mirror whenever light of only one of the two polarization directions is omnidirectionally reflected. In the other plots in Fig. 6 for higher and lower frequencies, the radiative-mode parts of $\Gamma_{x}$ are the sums of emission rates into both polarization directions.

\section{CONCLUSIONS, DISCUSSION, AND OUTLOOK}

A theory was set up for the multiple scattering of vector waves by parallel planes, thereby generalizing previous work for scalar waves to the more interesting but also more complicated case of light waves. Unlike for scalar waves, the Green function had to be regularized. This was accomplished by introducing a high-momentum cutoff. An effective scattering theory emerged with a nonzero $T$ matrix that no longer depends on the cutoff. The $T$ matrix and Green-function formalism turned out to be very convenient for the calculation of propagating and guided modes, as well as spontaneousemission rates, of finite photonic crystals of plane scatterers.

A nonabsorbing plane scatterer satisfies a separate optical theorem for $s$ - and $p$-polarized light. The radiative and guided modes of $s$-polarized light could be mapped onto modes for scalar waves. The $s$-polarized light has continuous modes, whereas $p$-polarized modes have discontinuities at the plane positions.

Throughout the paper, we have stressed the similarities and differences of optical properties of a plane scatterer or a crystal of plane scatterers as compared to the corresponding dielectric slab structures. It turns out that $p$-polarized waves differ more in the two cases than $s$-polarized waves. First, because propagating $p$-polarized modes are different in the two cases because the Brewster angle is $90^{\circ}$ for plane scatterers. Second, $p$-polarized guided modes in finite slab structures have no analogs for plane scatterers. This was also found in Ref. [15] for the single plane and in Refs. [15,20] for the infinite crystal.

Unlike for scalar waves, equidistant and identical plane scatterers cannot be an omnidirectional mirror for all vector waves. Such omnidirectional mirrors consisting of dielectric layers do exist. For layered media, at least three refractive 
indices are required in order to prevent complete transmission of $p$-polarized light in the Brewster-angle direction $[3,4,43]$.

Omnidirectional reflection is a property of a dielectric for external light sources. In this paper, omnidirectional reflection could be related to the emission properties of atomic light sources from within the finite crystals. The graphs of emission rates (Fig. 6) show that the emission by dipoles oriented parallel to the planes is affected much more strongly by the planes than emission by perpendicular dipoles. This is a characteristic of the plane-scatterer model, because the absence of $p$-polarized guided modes is responsible for much of the difference. In the frequency interval where $s$-polarized light is omnidirectionally reflected, all light that exits the crystal after a spontaneous-emission process will be $p$ polarized, irrespective of the orientation of the emitters. Still, the major fraction of the light will be emitted into guided modes and stay inside the crystal.

For low frequencies, the single-plane reflectivity is lower, emission rates are less affected by the crystal, and finite-size effects are appreciable also in the inner unit cells of the tenplane crystal. For higher frequencies, planes reflect light better and emission rates are more strongly modified. In the inner unit cells, the emission rates converge faster to the values of the unit cell of an infinite crystal. If the infinite crystal has larger variations in the emission rates inside a unit cell, then a smaller finite crystal is needed to converge to this result.

We argued that the guided modes will give rise to a peak in the frequency-dependent emission rate of a parallel dipole close to a plane. We also reasoned that the peak will shrink and shift to lower frequencies when either the effective thickness or the distance to the plane is increased. Indeed, the occurrence of a peak and its dependence on the effective thickness agree with numerical calculations on infinite crystals in Ref. [19]; some more numerical work is needed to corroborate our prediction of the distance dependence.

What other purposes can plane scatterers serve in the future? The finite photonic crystals that can be made with them have one-dimensional periodicity only, yet light propagation in all three dimensions for all polarization directions is properly taken into account. The number of planes can be chosen at will, and further advantages are that all optical modes and the complete Green tensor can be determined.

Our calculations can be extended to situations where not all planes are identical; or one could allow light absorption or gain in the planes by giving the effective thickness a complex value; the number of planes per unit cell could also be increased to more than one. Such calculations are possible in our formalism because the results (34) and (35) for the $T$ matrix were generalized in Eq. (A6) of the Appendix to planes chosen at arbitrary positions, each with a different $T$ matrix $\mathbf{T}_{\alpha}\left(\mathbf{k}_{\|}, \omega\right)$, in other words with a different effective thickness. The model can therefore also be used to study the effects of disorder in the positions or in the optical properties of the planes on the spontaneous-emission rates of embedded atoms.

It would also be interesting to study models of finite photonic crystals built up of nonparallel planes. This can be done, but numerically the model would become more involved, not so much because the planes have an overlap of measure zero, but rather because the plane representations for nonparallel planes will be different. Numerical calculations for nonparallel planes require the discretization of individual planes.

Apart from extending it, one can extract other interesting observables from the model. For example, the knowledge of the complete Green function makes it possible to calculate both far-field and near-field spectra of atoms embedded in the finite crystal. It would be interesting to study spectra near frequencies where the corresponding infinite crystal gives rise to a Van Hove singularity in the emission rates [20]. Crystals of plane scatterers can serve as a model environment to study the modification of several quantum optical processes when atoms are embedded in photonic crystals. Transient effects in the spontaneous-emission rates are but one example, thereby generalizing work done on a onedimensional cavity formed by two planes [44]. Calculations are underway that show photonic-crystal-induced modifications of cooperative atomic processes.

\section{ACKNOWLEDGMENTS}

We would like to thank Rudolf Sprik and Willem Vos for stimulating discussions. This work is part of the research program of the Stichting voor Fundamenteel Onderzoek der Materie, which was financially supported by the Nederlandse Organisatie voor Wetenschappelijk Onderzoek.

\section{APPENDIX: DERIVATION OF $N$ PLANE $T$ MATRIX}

The goal of this appendix is to derive the expressions (34) and (35) for the $N$-plane $T$ matrix $T^{(N)}$, by summing the infinite series in Eq. (12), where the $\mathbf{T}_{\alpha}$ are the $T$ matrices of single planes:

$$
\mathbf{T}_{\alpha}(\omega)=\frac{1}{(2 \pi)^{2}} \int d^{2} \mathbf{k}_{\|}\left|\mathbf{k}_{\|}, z_{\alpha}\right\rangle \mathbf{T}_{\alpha}\left(\mathbf{k}_{\|}, \omega\right)\left\langle z_{\alpha}, \mathbf{k}_{\|}\right|
$$

$\mathbf{T}_{\alpha}\left(\mathbf{k}_{\|}, \omega\right)$ are $3 \times 3$ tensors, defined in Eq. (28). First allow all planes to have different $\mathbf{T}_{\alpha}\left(\mathbf{k}_{\|}, \omega\right)$; furthermore, allow the parallel planes to have arbitrary (but all different) positions $z_{\alpha}$.

The first-order term in the expansion (12) is simply the sum of $\mathbf{T}_{\alpha}$ of Eq. (A1); the second-order term has the form

$$
\frac{1}{(2 \pi)^{2}} \int d \mathbf{k}_{\|} \sum_{\alpha, \beta=1}^{N}\left|\mathbf{k}_{\|}, z_{\alpha}\right\rangle \mathbf{T}_{\alpha}\left(\mathbf{k}_{\|} ; \omega\right) \cdot\left[\mathbf{D}\left(\mathbf{k}_{\|}, \omega\right)\right]_{\alpha \beta}\left\langle\mathbf{k}_{\|}, z_{\beta}\right|,
$$

where the dot denotes the inner product of $3 \times 3$ tensors. Furthermore, the property $\left\langle\mathbf{k}_{\|}, z\left|\mathbf{G}_{0}(\omega)\right| \mathbf{k}_{\|}^{\prime}, z^{\prime}\right\rangle$ $=(2 \pi)^{2} \delta^{2}\left(\mathbf{k}_{\|}-\mathbf{k}_{\|}^{\prime}\right) \mathbf{G}_{0}\left(\mathbf{k}_{\|}, z, z^{\prime}, \omega\right)$ of the free-space Green function was used. The matrix elements $\mathbf{D}_{\alpha \beta}$ of the matrix $\mathbf{D}$ are $3 \times 3$ tensors which are defined as

$$
\left[\mathbf{D}\left(\mathbf{k}_{\|}, \omega\right)\right]_{\alpha \beta} \equiv\left(1-\delta_{\alpha \beta}\right) \mathbf{G}_{0}\left(\mathbf{k}_{\|}, z_{\alpha}, z_{\beta}, \omega\right) \cdot \mathbf{T}_{\beta}\left(\mathbf{k}_{\|}, \omega\right) .
$$


The third-order term of the expansion (12) is as Eq. (A2), with $\mathbf{D}_{\alpha \beta}$ replaced by $\mathbf{D}_{\alpha \beta}^{2}=\sum_{\gamma=1}^{N} \mathbf{D}_{\alpha \gamma} \cdot \mathbf{D}_{\gamma \beta}$, that is, the square of the $N \times N$ matrix $\mathbf{D}$. Similarly, the fourth-order term features the cube of $\mathbf{D}$, and so on. By summing all orders, we have

$$
\mathbf{T}^{(N)}(\omega)=\frac{1}{(2 \pi)^{2}} \int d^{2} \mathbf{k}_{\|} \sum_{\alpha, \beta=1}^{N}\left|\mathbf{k}_{\|}, z_{\alpha}\right\rangle \mathbf{T}_{\alpha \beta}^{(N)}\left(\mathbf{k}_{\|}, \omega\right)\left\langle\mathbf{k}_{\|}, z_{\beta}\right|,
$$

where the $N$-plane $T$ matrix $\mathbf{T}_{\alpha \beta}^{(N)}\left(\mathbf{k}_{\|}, \omega\right)$ is given by the series

$$
\begin{aligned}
\mathbf{T}_{\alpha \beta}^{(N)}\left(\mathbf{k}_{\|}, \omega\right) & =\mathbf{T}_{\alpha} \cdot\left[\mathbf{I} \otimes \mathbf{I}_{N}+\mathbf{D}+\mathbf{D}^{2}+\mathbf{D}^{3}+\cdots\right]_{\alpha \beta} \\
& =\mathbf{T}_{\alpha} \cdot\left[\mathbf{I} \otimes \mathbf{I}_{N}-\mathbf{D}\right]_{\alpha \beta}^{-1} .
\end{aligned}
$$

Here, $\mathbf{I}_{N}$ is the $N \times N$ unit matrix and, as before, $\mathbf{I}$ is the 3 $\times 3$ unit tensor, so that $\left[\mathbf{I} \otimes \mathbf{I}_{N}\right]_{\alpha \beta}=\delta_{\alpha \beta} \mathbf{l}$. Now in the plane representation, the Green tensor has the form as derived in Sec. III A, and the single-plane $T$ matrices $\mathbf{T}_{\alpha}\left(\mathbf{k}_{\|}, \omega\right)$ have the simple form as defined in Eq. (28), with only $T_{\alpha}^{s S}$ and $T_{\alpha}^{v v}$ nonzero. It follows that $\mathbf{D}_{\alpha \beta}, \mathbf{D}_{\alpha \beta}^{2}$, and all higher powers of D have only three nonzero spatial components, namely, the $s s, v v$, and the $z v$ components. As a consequence, $\mathbf{T}_{\alpha \beta}^{(N)}\left(\mathbf{k}_{\|}, \omega\right)$ has only two nonzero spatial components for all plane indices $\alpha$ and $\beta$, namely, the ss and $v v$ component:

$$
\mathbf{T}_{\alpha \beta}^{(N)}\left(\mathbf{k}_{\|}, \omega\right)=\left(\begin{array}{ccc}
T_{\alpha}^{s s}\left[\mathbf{I}_{N}-\mathbf{D}^{s s}\right]_{\alpha \beta}^{-1} & 0 & 0 \\
0 & T_{\alpha}^{v v}\left[\mathbf{I}_{N}-\mathbf{D}^{v v}\right]_{\alpha \beta}^{-1} & 0 \\
0 & 0 & 0
\end{array}\right) .
$$

This generalizes the result of Eq. (28) for the single-plane $T$ matrices $\mathbf{T}_{\alpha}$, where the same two components were found to be nonzero. The generalization is from one plane to $N$ parallel planes placed at arbitrary positions, each plane possibly with a different effective thickness. In the special case considered in the main text that all planes are identical, in other words if all $\mathbf{T}_{\alpha}\left(\mathbf{k}_{\|}, \omega\right)$ are equal, then Eq. (A6) simplifies into Eq. (35).
[1] Photonic Crystals and Light Localization in the 21st Century, edited by C. M. Soukoulis (Kluwer Academic, Dordrecht, 2001).

[2] E. Yablonovitch, Phys. Rev. Lett. 58, 2059 (1987); S. John, ibid. 58, 2486 (1987).

[3] Y. Fink, J.N. Winn, S. Fan, C. Chen, J. Michel, J.D. Joannopoulos, and E.L. Thomas, Science 282, 1679 (1998).

[4] C. Hooijer, D. Lenstra, and A. Lagendijk, Opt. Lett. 25, 1666 (2000).

[5] M. Wubs and A. Lagendijk, Phys. Rev. E 65, 046612 (2002).

[6] G. Kurizki and A.Z. Genack, Phys. Rev. Lett. 61, 2269 (1988); S. John and J. Wang, Phys. Rev. B 43, 12772 (1991); S. Bay, P. Lambropoulos, and K. Mølmer, Phys. Rev. A 55, 1485 (1997).

[7] R. Sprik, B.A. van Tiggelen, and A. Lagendijk, Europhys. Lett. 35, 265 (1996).

[8] K. Busch and S. John, Phys. Rev. E 58, 3896 (1998).

[9] G. Colas des Francs, C. Girard, J.-C. Weeber, C. Chicane, T. David, A. Dereux, and D. Peyrade, Phys. Rev. Lett. 86, 4950 (2001); G. Colas des Francs, C. Girard, J.-C. Weeber, and A. Dereux, Chem. Phys. Lett. 345, 512 (2001).

[10] K. Busch C. R. Physique 3, 53 (2002).

[11] T. Suzuki and P.K.L. Yu, J. Opt. Soc. Am. B 12, 570 (1995).

[12] Z.-Y. Li, L.-L. Lin, and Z.-Q. Zhang, Phys. Rev. Lett. 84, 4341 (2000); Z.-Y. Li and Y. Xia, Phys. Rev. A 63, 043817 (2001).

[13] S. John and J. Wang, Phys. Rev. Lett. 64, 2418 (1990); S. John and T. Quang, Phys. Rev. A 50, 1764 (1994); S. John and T. Quang, Phys. Rev. Lett. 74, 3419 (1995); E. Paspalakis, N.J. Kylstra, and P.L. Knight, Phys. Rev. A 60, R33 (1999); G.M. Nikolopoulos and P. Lambropoulos, ibid. 61, 053812 (2000).

[14] J.P. Dowling and C.M. Bowden, Phys. Rev. A 46, 612 (1992).

[15] T.J. Shepherd, P.J. Roberts, and R. Loudon, Phys. Rev. E 55, 6024 (1997).

[16] P.M. Visser and G. Nienhuis, Opt. Commun. 136, 470 (1997).
[17] I. Alvarado-Rodriguez, P. Halevi, and J.J. SanchezMondragón, Phys. Rev. E 59, 3624 (1999).

[18] J.R. Zurita-Sánchez and P. Halevi, Phys. Rev. E 61, 5802 (2000).

[19] I. Alvarado-Rodriguez, P. Halevi, and A.S. Sánchez, Phys. Rev. E 63, 056613 (2001); 65, 039901(E) (2002).

[20] J.R. Zurita-Sánchez, A.S. Sánchez, and P. Halevi, Phys. Rev. E 66, 046613 (2002).

[21] K.H. Drexhage, J. Lumin. 1-2, 693 (1970).

[22] S. Haroche, in Fundamental Systems in Quantum Optics, Proceedings of the Les Houches Summer School, Session 53, edited by J. Dalibard, J.-M. Raimond, and J. Zinn-Justin (NorthHolland, Amsterdam, 1992), p. 767.

[23] P. W. Milonni, The Quantum Vacuum: An Introduction to Quantum Electrodynamics (Academic Press, San Diego, 1994).

[24] J. Eschner, Ch. Raab, F. Schmidt-Kaler, and R. Blatt, Nature (London) 413, 495 (2001).

[25] A. Beige, J. Pachos, and H. Walther, Phys. Rev. A 66, 063801 (2002).

[26] M.S. Tomaš, Phys. Rev. A 51, 2545 (1995).

[27] A. Yariv and P. Yeh, Optical Waves in Crystals (Wiley, New York, 1984).

[28] J.M. Bendickson, J.P. Dowling, and M. Scalora, Phys. Rev. E 53, 4107 (1996).

[29] D.J. Griffiths and C.A. Steinke, Am. J. Phys. 69, 137 (2001).

[30] A.A. Asatryan, K. Busch, R.C. McPhedran, L.C. Botten, C.M. de Sterke, and N.A. Nicorovici, Phys. Rev. E 63, 046612 (2001); Waves Random Media 13, 9 (2003).

[31] O.J.F. Martin, C. Girard, D.R. Smith, and S. Schultz, Phys. Rev. Lett. 82, 315 (1999).

[32] M. Doosje, B.J. Hoenders, and J. Knoester, Opt. Commun. 206, 253 (2002).

[33] A. Gonis and W. H. Butler, Multiple Scattering in Solids (Springer, New York, 2000). 
[34] T. Søndergaard and B. Tromborg, Phys. Rev. B 66, 155309 (2002).

[35] J. Schwinger, L.L. DeRaad, Jr., and K.A. Milton, Ann. Phys. (N.Y.) 115, 23 (1978).

[36] P. de Vries, D.V. van Coevorden, and A. Lagendijk, Rev. Mod. Phys. 70, 447 (1998).

[37] R. Loudon, The Quantum Theory of Light (Clarendon Press, Oxford, 1983).

[38] H.P. Urbach and G.L.J.A. Rikken, Phys. Rev. A 57, 3913 (1998).
[39] R.J. Glauber and M. Lewenstein, Phys. Rev. A 43, 467 (1991).

[40] G.S. Agarwal, Phys. Rev. A 12, 1475 (1975); J.M. Wylie and J.E. Sipe, ibid. 30, 1185 (1984).

[41] L. Knöll, S. Scheel, and D.-G. Welsch, in Coherence and Statistics of Photons and Atoms, edited by J. Perrina (Wiley, New York, 2001).

[42] S.M. Barnett, B. Huttner, R. Loudon, and R. Matloob, J. Phys. B 29, 3763 (1996).

[43] J. Lekner, J. Opt. A, Pure Appl. Opt. 2, 349 (2000).

[44] O. Jedrkiewicz and R. Loudon, Phys. Rev. A 60, 4951 (1999). 